\title{
ANNOTATED CHECKLIST OF CAPITELLIDAE (ANNELIDA, POLYCHAETA) FROM THE IBERIAN PENINSULA, CHAFARINAS, BALEARIC AND CANARY ISLANDS
}

\author{
M. El Haddad*, R. Capaccioni Azzati \& A. M. García-Carrascosa
}

\begin{abstract}
M. El Haddad, R. Capaccioni Azzati \& A. M. García-Carrascosa. 2013. Annotated checklist of Capitellidae (Annelida, Polychaeta) from the Iberian Peninsula, Chafarinas, Balearic and Canary Islands. Grael/sia, 69(1): 97-116.

The present annotated checklist has been elaborated after revision of preserved materials (from the Museo Nacional de Ciencias Naturales, Madrid and the reference collection of the Marine Biology Laboratory of the University of Valencia) and published literature related to capitellids from the Iberian Peninsula, Chafarinas, Balearic and Canary Islands. Twenty-four species and subspecies belonging to thirteen genera are recognized as valid taxa. With this checklist we include some taxonomic details and information on distribution at both global and regional levels. This work includes the setal formula and diagrammatic representation of capitellid taxa treated in this study.
\end{abstract}

Key words: Polychaeta; Capitellidae; taxonomy; Iberian Peninsula; Chafarinas Islands; Balearic Islands; Canary Islands.

\section{RESUMEN}

M. El Haddad, R. Capaccioni Azzati \& A. M. García-Carrascosa. 2013. Listado taxonómico comentado de los Capitélidos (Annelida, Polychaeta) de la península lberica, islas Chafarinas, Baleares y Canarias. Graellsia, 69(1): 97-116.

Se ha elaborado un listado taxonómico comentado de los capitélidos de la Península Ibérica, Islas Chafarinas, Baleares y Canarias a partir de la revisión de materiales (Museo Nacional de Ciencias Naturales, Madrid y colección de referencia del Laboratorio de Biología Marina de la Universidad de Valencia) y de la bibliografía publicada referente a este grupo. Se reconocen como taxones válidos 24 especies y subespecies pertenecientes a 13 géneros. En esta lista se incluyen para cada especie algunos detalles taxonómicos e información sobre su distribución geográfica tanto a nivel mundial como regional. Asimismo, se aporta la fórmula setal y el diagrama gráfico para los taxones contemplados en este estudio.

Palabras clave: Polychaeta; Capitellidae; taxonomía; Península lbérica; Islas Chafarinas; Islas Baleares; Islas Canarias.

Departamento de Zoología, Laboratorio de Biología Marina,Universitat de València, Facultad de Ciencias Biológicas, C/ Dr Moliner 50, 46100, Burjassot, Valencia, España. e-mail: Mustapha.Haddad@uv.es

* Corresponding author: Mustapha.Haddad@uv.es 


\section{Introduction}

Capitellids superficially resemble earthworms due to the absence of appendages in the head and the presence of long cylindrical bodies with poorly developed parapodia (chaeta appear to arise directly from the body wall). They range in length from $1-2 \mathrm{~mm}$ to more than $90 \mathrm{~cm}$. The body is divided into a thorax (anterior region) and abdomen (posterior region). There are two principal types of setae: the capillaries, generally restricted to anterior thoracic setigers and the hooded hooks, usually present in posterior thoracic and abdominal setigers.

Because of their simplicity, Capitellids are among the most difficult groups to identify (Ewing, 1984). The number of thoracic setigers and the setal arrangements on the thoracic region are the most important taxonomic characters within this family. However, significant variations in the thoracic setal formula may be observed in smaller specimens (Ewing, 1984). For example, some anterior segments of Heteromastus filiformis may possess hooks in young individuals, being progressively replaced by capillaries with age (Fredette, 1982). Some taxa have posterior thoracic setigers and anterior abdominal segments with mixed fascicles of capillaries and hooded hooks making the separation between the thorax and abdomen difficult to appreciate. Another problem in the taxonomy of Capitellids is the use of the presence of "branchiae" as a taxonomic character because they can be completely retracted in preserved material (Hutchings, 2000) or also restricted to posterior abdominal segments (e.g. Pseudomastus), knowing that it is difficult to collect complete specimens of these worms due to their fragility.

The capitellids are one of the first recognized groups of polychaetes. Capitella capitata (Fabricius, 1780) was the first capitellid to be described and was placed in the clitellate genus Lumbricus. Grube (1862) erected "Capitellacea" to include taxa Capitella, Dasybranchus, and Notomastus; since then capitellids were recognized as a separate family within the Polychaeta.

The first publications referring to capitellid species reported from the study area are those of Langerhans (1881) on Notomastus latericeus, in the Canary Islands, and Roule (1896) on Notomastus agassizii in the Iberian Peninsula. Many works, including partial lists of this group, were published; among the most interesting ones are Rioja (1931), Ibáñez (1973a), Campoy (1982), Ariño (1987),
Parapar et al. (1996) and Núñez et al. (2005). Over the last two decades, the largest number of studies, wholly or partially dedicated to the polychaetes of the Iberian Peninsula, were carried out in the North Atlantic, in the Bay of Biscay (e.g. Aguirrezabalaga \& Ceberio, 2003, 2005, 2006; Aguirrezabalaga et al., 2001, 2002, 2006; Garmendia et al., 2003; Martínez \& Adarraga, 2001; Martínez et al., 2005, 2006, 2007a), Cantabrian Sea (Serrano, 2002; Serrano et al., 2006) and Galicia (e.g. Parapar, 1991; Moreira, 2002; Moreira et al., 2003, 2006; Cacabelos, 2005; Quintas, 2005; Cacabelos et al., 2008a, 2008b; Lourido et al., 2008; Parapar \& Moreira, 2009; Parapar et al. 2009).

The aim of the present work is to compile most of the available data about capitellid species reported from the Iberian Peninsula, Chafarinas, Balearic and Canary Islands in order to provide a checklist of the valid species therein recognized.

This checklist updates and completes the knowledge of capitellids in the study area by providing the available synonyms, as well as information on species distribution.

\section{Material and methods}

We defined ten broad biogeographical sectors for the whole studied area, based on the different geomorphologic and hydrographic characteristics (Fig. 1): (A) Catalonia, from Cape Creus to Ebro Delta; (B) Valencia coast and Balearic Islands, from Castellon to Cape Palos; (C) Murcia and Almería from Cape Palos to Motril; (D)Alboran Sea, from Motril to the Strait of Gibraltar including Chafarinas Islands; (E) Gulf of Cádiz, from the Strait of Gibraltar to Cape St. Vincent; (F) Portugal, from Cape St. Vincent to Miño River; (G), Galicia; (H) Cantabrian Sea; (I) Bay of Biscay and (J), Canary Islands.

The study was carried out during the review of preserved materials, from the Museo Nacional de Ciencias Naturales, Madrid (MNCN) and the reference collection of the Marine Biology Laboratory of the University of Valencia, and published literature related to this group within the "Iberian Fauna" project as part of the research project "Biodiversity Programme of the Iberian Peninsula", which was supported by The General Direction of Superior Studies (DGES) of the Spanish Ministry of Science and Technology. 

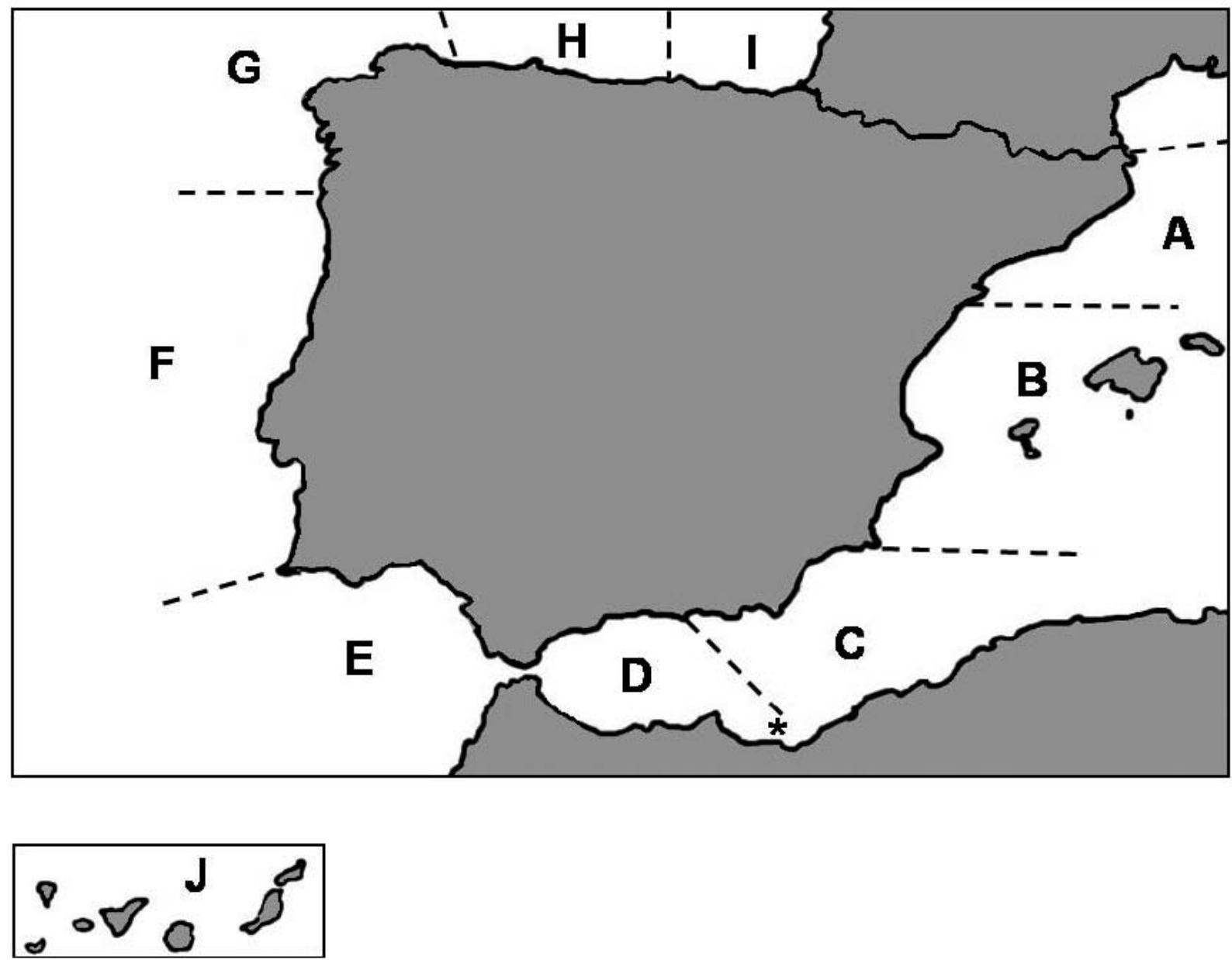

Fig. 1.- Map of the study area. A, Catalonia; B, Valencia coast and Balearic Islands; C, Murcia and Almería; D, Alboran Sea and Chafarinas Islands (*); E, Gulf of Cádiz; (F) Portugal; G, Galicia; H, Cantabrian Sea; I, Bay of Biscay and J, Canary Islands.

Fig. 1.- Mapa de la zona de estudio. A, Cataluña; B, Costas de Valencia e Islas Baleares; C, Murcia y Almería; D, Mar de Alboran e Islas Chafarinas (*); E, Golfo de Cádiz; (F) Portugal; G, Galicia; H, Mar Cantábrico; I, Golfo de Vizcaya y J, Islas Canarias.

The checklist presented below comprises a total of 24 species and subspecies with representatives from 13 genera. Taxa identified to genus and confirmed misidentified species were not taken into account in this study. The capitellids are arranged alphabetically by genera, as are species within genera. The obvious orthographic errors in the names of the taxa have been corrected. Critical comments are given only for a few taxa, mainly in order to clarify their nomenclature or to give our opinion on specially difficult cases.
We provided in Appendix the adult setal formula of peristomium and thorax (Day, 1973) and diagrammatic representations of peristomium, prostomium, thorax and some abdominal segments of these capitellids, modified from Hartman (1947), Amaral (1980), Ewing (1984) and Gravina \& Somaschini (1990). Taxa are also organized alphabetically. Both representations are given for genera or species when necessary. 


\section{Results}

Family CAPITELLIDAE Grube, 1862

Capitellidae Grube, 1862. Arch. Naturgesch., 28: 366

Genus Capitella Blainville, 1828

Capitella Blainville, 1828. Dict. Sci. Nat., 57: 443

TYPE SPECIES: Lumbricus capitatus Fabricius, 1780

Lumbriconais Oersted, 1842. Naturhist. Tidsskrift, 4: 132

Valla Johnston, 1865. Cat. Brit. non paras. Worms Brit. Mus.: 67 Capitomastus Eisig 1887. Fauna Flora Golf. Neapel, 16: 857 Capitellides Mesnil, 1897. Zool. Anz., 20: 441

Isomastus Gravier, 1911. Bull. Mus. Hist. nat. Paris, 17: 313

Capitella capitata (Fabricius, 1780) sensu latu

Lumbricus capitatus Fabricius, 1780. Fauna Groenl.: 279. Type locality/origin: SW Greenland.

Ancistria acuta Verrill, 1874a. Am. J. Sci. 7: 505

Capitella fabricii Blainville, 1828. Diet. Sci. Nat., 57: 443

Lumbricus canalium Nardo, 1847. Prosp. Fauna mar. Veneto: 11

Lumbriconais marina Oersted, 1842. Kroyer's Naturh. Tidsskr., 4: 132

Lumbricus littoralis Johnston, 1827. Zool. Journ., 3: 328

Capitella capitata: Beneden, 1857. Bull. Acad. Roy. Bel., 3: 137

Valla ciliata Johnston, 1865. Cat. Worms. Brit. Mus.: 68

GLOBAL DISTRIBUtion: cosmopolitan for this species complex.

REGIONAL DisTRIBUTION: All sectors.

REMARKS: Capitella capitata was initially considered a single cosmopolitan species but was later shown to be a large complex of more than 50 cryptic sibling species (Méndez et al., 2000; Méndez, 2006). The type material of $C$. capitata is lost. Recently Blake (2009) designated a neotype from Mârmorilik (West Greenland) and gave a redescription for this species to establish a baseline for a future revision of the Capitella species complex. Notice that current definitions of $C$. capitata based on the work of Eisig (1887) from the Gulf of Naples and supported by Hartman (1947) and Warren (1976) refer to other species that differ significantly from the Greenland specimens (Blake, 2009). Greenland specimens have a narrow peristomial ring retracted slightly into the first setiger (preserved material) that may become difficult to see and may be easily interpreted as being fused with the first thoracic setiger (Warren, 1991).

Capitella giardi (Mesnil, 1897)

Capitellides giardi Mesnil, 1897. Zool. Anz., 20: 442. Type locality/origin: "côtes de la Manche" (France, NE Atlantic)

Capitomastus giardi (Mesnil): Hartmann-Schröder, 1971. Tierwelt Dtschl., 58: 896
Capitella giardi (Mesnil): Warren, 1976. J. Zool., Lond., 180: 205

GlobAL DISTRIBUTION: From northeastern Atlantic, North Sea and Baltic Sea to the Galician coast. Mediterranean Sea in Greece (Simboura \& Nicolaidou, 2001), France (Carpine, 1970). Doubtful records in Italy (Gravina \& Somaschini, 1990; Castelli et al., 1995).

Regional distribution: G (La Coruña and Lugo: García et al., 1979. Pontevedra: Villalba \& Viéitez, 1985).

Capitella minima Langerhans, 1880

Capitella minima Langerhans, 1880. Zeits. wiss. Zool. 34: 99. Type locality/origin: Madeira (NE Atlantic)

Capitomastus minimus (Langerhans): Eisig 1887. Fauna Flora Golf. Neapel, 16: 857

GLOBAL DISTRIBUtion: Eastern Atlantic from British Channel to Morocco (Rullier \& Amoureux, 1970), Madeira (Langerhans, 1880) and Canary Islands. Western Atlantic in Brazil (Rizzo \& Amaral, 2001). Mediterranean Sea. Black Sea (Rullier, 1963). Red Sea (Ben-Eliahu, 1976), Madagascar (Thomassin, 1970) and the Andaman Sea (Green, 2002).

Regional Distribution:

A (Rosas Bay: Desbruyères et al., 1972. Barcelona: Sardá, 1986. Cabo de Creus: Alós, 1988; Alós \& Pereira, 1989).

C (Mar Menor: Capaccioni, 1983).

D (Málaga: Acero \& San Martín, 1986. Algeciras Bay: Sardá, 1984. Chafarinas Islands: López, 1995; Tena, 1996). Ceuta (Parapar et al., 1993).

E (Cádiz: Ibáñez, 1973a. Mouth of Piedras River: López Serrano, 1999).

G (Pontevedra: Viéitez, 1979; Muros Ria: LópezJamar, 1981; Tenore et al., 1984. Arosa Ria: LópezJamar, 1982; Tenore et al., 1984. Vigo Ria: Viéitez \& López Cotelo, 1982. Corcubión Ria and Cabo Finisterre: Tenore et al., 1984).

I (Urumea Ría: Ibáñez et al., 1984).

H (Bahía de Santander: López-Cotelo et al., 1982).

J (Lanzarote, Fuerteventura, Gran Canaria, Tenerife and El Hierro Islands: Brito, 1999).

Genus Dasybranchus Grube, 1850

Dasybranchus Grube, 1850 Arch. Naturgesch., 16 (1), 324

TYPE SPECIES: Dasymallus caducus Grube, 1846

Dasymallus Grube, 1846. Arch. Naturgesch., 12 (1): 166

Branchoscolex Schmarda, 1861. N. wirbell. Thiere, 1 (2): 59

Dasybranchus caducus (Grube, 1846)

Dasymallus caducus Grube, 1846. Arch. Naturgesch.,12 (1): 166. Type locality/origin: "Küste des Mittelmeers" (Mediterranean Sea) 
Dasybranchus caducus (Grube): Grube, 1850. Arch. Naturgesch., $16(1), 324$

Dasybranchus cirratus Grube, 1867. Zool.Theil, 2 (3): 28

Dasybranchus umbrinus Grube, 1878. Mém. Acad. Imp. Sci. St.-Pétersbg., (7), 25: 189

Notomastus roseus Langerhans, 1880. Z. Wiss. Zool, 34 (1): 99

GlobAL Distribution: Circumglobal in warm and warm-temperate seas.

REGIONAL DISTRIBUTION:

A (Rosas Bay: Desbruyères et al., 1972. Blanes: Camp, 1976; Campoy \& Jordana, 1978; Campoy, 1982).

B (Columbretes Islands: Campoy, 1979. Islas Baleares: Sardá, 1991).

D (Off Alboran Island at l $=35^{\circ} 50^{\prime} \mathrm{N}$; L $=003^{\circ} 16,5^{\prime} \mathrm{W}$ :

Bellan, 1959. Fuengirola: San Martín et al., 1982).

F (Faro: Nogueira de Carvalho, 1929).

G (Ría de La Coruña: García Álvarez et al., 1993)

H (Santander: Rioja, 1917).

J (Tenerife: Núñez et al., 2005).

\section{Dasybranchus gajolae Eisig, 1887}

Dasybranchus gajolae Eisig, 1887. Fauna Flora Golf. Neapel, 16: 828. Type locality/origin: Gulf of Naples (Italy, Mediterranean Sea)

Dasybranchus caducus (Grube): Claparède, 1864. Mém. Soc. Phys. Hist. Nat. Genève, 17(2): 516 (non Grube, 1846)

GLOBAL DISTRIBUTION: Eastern Atlantic from Brittany (Glemarec, 1969) to the Strait of Gibraltar (Sardá, 1984), Azores and Madeira (Fauvel, 1927). Mediterranean Sea and Marmara Sea (La Greca, 1949; Rullier, 1963).

REgional Distribution:

A (Rosas Bay: Desbruyères et al., 1972. Cabo de Creus: Alós, 1988; Alós \& Pereira, 1989. Barcelona: Méndez \& Cardell, 1996. La Merenguera canyon, off Barcelona: Ramírez-Llodra et al., 2008)

B (Mallorca: Rioja, 1935)

C (Murcia: San Martín \& Viéitez, 1984)

D (Algeciras Bay: Sardá, 1984. Chafarinas Islands: López, 1995; Tena, 1996)

E (Ibáñez, 1973a)

F (Faro: Fauvel, 1914)

G (?Galician slope: Amoureux, 1972)

I (Martínez et al., 2005, 2006, 2007a, 2007b)

Genus Heteromastus Eisig, 1887

Heteromastus Eisig, 1887. Fauna Flora Golf. Neapel, 16: 835 TYPE SPECIES: Capitella filiformis Claparède, 1864

Ancistria Quatrefages, 1865. Hist. Nat. Annelés Mar Eau Douce, 2: 252

? Areniella Verrill, 1874b. Proc. Am. Assoc. Adv. Sci., [1873], 22: 386
Heteromastus filiformis (Claparède, 1864)

Capitella filiformis Claparède, 1864. Mém. Soc. Phys. Hist. Nat. Genève, 17(2): 509. Type locality/origin: Port-Vendres (France, Mediterranean Sea)

Ancistria minima Quatrefages, 1865. Hist. Nat. Annelés Mar Eau Douce, 2: 252

Notomastus filiformis Verrill, 1873. Rep. U.S. Comm. Fish., 1: 611

? Areniella filiformis Verril, 1874b. Proc. Am. Assoc. Adv. Sci., [1873], 22: 386 (see Hutchings \& Rainer, 1981)

Notomastus laevis Webster, 1886. Annu. Rep. New York Mus. Nat. Hist., 39: 152

Heteromastus filiformis (Claparède, 1864): Eisig, 1887. Fauna Flora Golf. Neapel, 16: 839

Global Distribution: Cosmopolitan (Hutchings \& Rainer, 1981).

REGIONAL DISTRIBUTION: All sectors except “J”.

REMARKS: Hutchings \& Rainer (1981) designated the neotype for this species from Egypt. Unfortunately, they did not give any drawings and their diagnosis includes some typographical errors (Green, 2002).

Genus Leiocapitella Hartman 1947

Leiocapitella Hartman, 1947. Allan Hancock Pacif. Exped., 10: 437

TYPE SPECIES: Leiocapitella glabra Hartman, 1947

Leiocapitella glabra Hartman 1947

Leiocapitella glabra Hartman, 1947. Allan Hancock Pacif. Exped., 10: 438. Type locality/origin: Espiritu Santo Island, Gulf of California (E Pacific)

Mastobranchus dollfusi Fauvel, 1936. Mém. Soc. Sci. Nat. Maroc, 43: 81 (see remarks)

Leiocapitella dollfusi (Fauvel): Bellan, 1964. Rec. Trav. St. Mar. End., 49(33): 145

Global Distribution: Northern Gulf of Mexico (Ewing 1984), southern California and Gulf of California (Hartman 1947, Hernández-Alcántara \& Solís-Weiss, 1998). Eastern Atlantic from Biscay Bay (Martínez et al., 2006) to Morocco (Fauvel, 1936) and Congo (Kirkegaard, 1959). Mediterranean Sea. Green (2002) recorded Leiocapitella near glabra from the Indian Ocean.

REGIONAL DISTRIBUTION:

A (Rosas Bay: Desbruyères et al., 1972. Barcelona: Méndez \& Cardell, 1996)

G (Galician slope: Amoureux, 1972)

H (Cantabrian slope: Amoureux, 1973. ?Banco Le

Danois: Amoureux, 1974)

I (Biscay: Martínez et al., 2006)

REMARKS: Mastobranchus dollfusi described by Fauvel (1936) was transferred to the genus Leiocapitella 
by Bellan (1964) as Leiocapitella dollfusi. Ben-Eliahu \& Fiege (1995) considered this species as a synonym of Leiocapitella glabra.

Genus Leiochrides Augener, 1914

Leiochrides Augener, 1914. Fauna Südwest-Aust. 5(1): 60. TYPE SPECIES: Leiochrides australis Augener, 1914

Leiochrides cf. africanus Augener, 1918

Leiochrides africanus Augener, 1918: 472. Type locality/origin: Setté Cama, Brazzaville Congo (SE Atlantic).

Global Distribution: Tropical western Africa from Ghana to Angola (Augener, 1918) and South Africa in Cape and Natal (Day, 1967). Madagascar (Thomassin, 1970). ?Red Sea (Wehe \& Fiege, 2002). Doubtful record in Canary Islands.

Regional Distribution: J (?Lanzarote Island: Núñez et al., 2005)

Genus Mastobranchus Eisig, 1887

Mastobranchus Eisig, 1887. Fauna Flora Golf. Neapel, 16: 831

Mastobranchus trinchesii Eisig, 1887

Mastobranchus trinchesii Eisig, 1887. Fauna Flora Golf. Neapel, 16: 833. Type locality/origin: Gulf of Naples (Italy, Mediterranean Sea).

Global Distribution: Mediterranean Sea, Indian Ocean (Thomassin, 1970) and eastern Atlantic in Liberia (Kirkegaard, 1959).

Regional distribution: A (Rosas Bay: Desbruyères et al., 1972. Barcelona: Méndez \& Cardell, 1996. Els Alfacs Bay: Martín et al., 1993, 2000; Brito et al., 2005)

Genus Mediomastus Hartman, 1944

Mediomastus Hartman, 1944. Allan Hancock Pac. Exped., 10: 264

TYPE SPECIES: Mediomastus californiensis Hartman, 1944.

Capitita Hartman, 1947. Allan Hancock Pacif. Exped., 10: 408

Mediomastus capensis Day, 1961

Mediomastus capensis Day, 1961. J. Linn. Soc. Lond. Zool., 44(299): 518. Type locality/origin: Saldanha Bay, South Africa.

Global Distribution: South Africa (Day, 1967) and Mozambique (Hartmann-Schröder, 1974). Portugal (Carvalho et al., 2001) and Mediterranean Sea in
Tyrrhenian and Ionian Seas (Gravina \& Somaschini, 1988, 1990; Lanera \& Gambi, 1993; Giangrande \& Gambi, 1986). Chafarinas Islands. Recorded with doubt in Catalonia (Desbruyères et al., 1972).

REGIONAL DISTRIBUTION:

A (?Rosas Port: Desbruyères et al., 1972)

D (Port of Isabel II Island: López, 1995)

G (Sado estuary: Carvalho et al., 2001)

Mediomastus fragilis Rasmussen, 1973

Mediomastus fragilis Rasmussen, 1973. Ophelia (11): 115. Type locality/origin: Isjeford (Kattegat)

Heteromastus filiformis (Claparède): Rasmussen, 1956, Biol. Meddr., 23(1): 64 (non Claparède (1864))

GLOBAL DISTRIBUTION: Northeastern Atlantic from the Shetlands (Warren, 1979) and Danish coast (Rasmussen, 1973), to Iberian Atlantic coasts. Mediterranean Sea.

REgiONAL DisTRIBUTION:

A (Cabo de Creus: Alós, 1988; Alós \& Pereira, 1989. Blanes: Martín, 1986; Sardá et al., 1995. Barcelona: Méndez \& Cardell, 1996. Els Alfacs Bay: Capaccioni, 1987; Sardá et al., 1995; Martín et al., 2000)

B (Outer Valencia Port: Tena, 1992)

C (Mar Menor: Capaccioni, 1983; Capaccioni Azzati, 1985)

D (Chafarinas Islands: Torres-Gavilá, 2008)

E (Mouth of Piedras River: López Serrano, 1999)

F (Aveiro Ria: Cunha \& Ravara, 2003)

G (Pontevedra: Planas, 1986)

H (Santander Bay: Lastra, 1991)

I (Zumaya Ria: Aguirrezabalaga et al., 1986. Bidasoa estuary: Garmendia et al., 2003. Continental shelf and upper margin of the continental slope of Guipuzcoa: Martínez \& Adarraga 2001; Martínez et al., 2005, 2007a,b.).

Genus Neopseudocapitella Rullier \& Amoureux, 1979 Neopseudocapitella Rullier \& Amoureux, 1979. Ann. Inst. Océanogr., 55 (Suppl.): 185

TYPE SPECIES: Neopseudocapitella brasiliensis Rullier \& Amoureux, 1979.

Neopseudocapitella brasiliensis Rullier \& Amoureux, 1979 Neopseudocapitella brasiliensis Rullier \& Amoureux, 1979. Ann. Inst. Océanogr., 55 (Suppl.): 185. Type locality/origin: (Brasil, SW Atlantic).

GlobAL DISTRIBUTION: Southwestern Atlantic in Brazil (Rullier \& Amoureux, 1979). Red Sea (Amoureux, 1983a). Adriatic (Amoureux, 1983b; Zavodnik et al., 1985) and Aegean Sea (Arvanitidis, 2000; Simboura \& Nicolaidou, 
2001). Mediterranean Iberian Peninsula and Chafarinas Islands.

REGIONAL DISTRIBUTION:

A (Els Alfacs Bay: Capaccioni, 1987; CapaccioniAzzati et al., 1992)

B (Sierra Helada: Villarroya, 2005)

D (Chafarinas Islands: Torres-Gavilá, 2008)

Genus Notomastus Sars, 1851

Notomastus Sars, 1851. Nyt Mag. For Naturvid. 6: 199

TYPE SPECIES: Notomastus latericeus Sars, 1851

Arenia Quatrefages, 1865. Hist. Nat. Annelés Mar Eau Douce, 2: 249

Sandanis Kinberg, 1867. Öfvers. K. Vetensk. Akad. Förh, 22 (4): 343

Eisigella Gravier, 1901. Bull. Mus. Hist. Nat. Paris, 7: 402

? Rashgua Wesenberg-Lund, 1949. Danish Sci. Invest. Iran, 4: 336

? Paraleiocapitella Thomassin, 1970. Rec. Trav. St. Mar. End., 10: 86

? Dodecaseta McCammon \& Stull, 1978. Bull. So. Calif. Acad. Sci. 77(1): 40

REMARKS: Ewing (1982), considered Rashgua Wesenberg-Lund (1949) and Paraleiocapitella Thomassin (1970) as synonyms of Notomastus. Additionally, Blake (2000) proposed to include Dodecaseta as another synonym of Notomastus. According to Green (2002), these actions would expand the diagnosis of Notomastus to what would become an unrecognizable genus having more generic variability than any another described genus of capitellids. For this reason Green (2002) adopted the conservative view without recognizing the synonyms of Notomastus given by Ewing (1982).

Notomastus aberans Day, 1957

Notomastus aberans Day, 1957. Ann. Natal Mus., 14: 105. Type locality/origin: "Kosy Bay, Inhaca Island, Morrumbene Estuary" (South Africa, Indian Ocean).

Global Distribution: South Africa (Day, 1961), tropical West Indian Ocean (Day, 1957) and Madagascar (Day, 1967). Mediterranean Sea.

\section{REgIONAL DISTRIBUTION:}

A (Els Alfacs Bay: Capaccioni-Azzati, 1988)

B (Guardamar del Segura: Torres-Gavilà, 1989. Valencia Port: El Haddad et al., 2007. Javea Port. Playa Levante, Benidorm: El Haddad, 2004)

D (Chafarinas Islands: Torres-Gavilá, 2008)

REMARKS: Notomastus aberans is considered as an established alien species in the Mediterranean Sea (Zenetos et al., 2005).
Notomastus agassizii McIntosh, 1885

Notomastus agassizii McIntosh, 1885. Rep. Sci. Results Voyag. Challenger, Zool., 12: 389. Type locality/origin: Off New York (NW Atlantic)

Global distribution: Amphi-Atlantic. Off New York (McIntosh, 1885). Bay of Biscay (Roule, 1896). ?Cape Verde (McIntosh, 1885).

Regional Distribution: H, I: $\left(\mathrm{l}=45^{\circ} 37^{\prime} \mathrm{N}\right.$; $\mathrm{L}=6^{\circ} 21^{\prime}$ $\mathrm{W}$ at $1410 \mathrm{~m}$ : Roule, 1896)

REMARKS: Notomastus agassizii is a poorly-defined species. Original description gived by McIntosh (1885) is incomplete without any clear taxonomic boundary with other Notomastus species. Up to now, there is no additional taxonomic work to complete its diagnoses.

\section{Notomastus exsertilis Saint-Joseph, 1906}

Notomastus exsertilis Saint-Joseph, 1906. Ann. Sc. Nat. Zool., (9), 3: 169. Type locality/origin: Bay of Saint-Jean-de-Luz (France, NE Atlantic)

? Dasyhranchus var. Saint-Joseph 1898. Ann. Sci. Nat., ZooL, (8), 5: 391

Global Distribution: Eastern Atlantic from the French coast (Fauvel, 1927), Galicia (Amoureux, 1972) and Bay of Biscay (Campoy, 1982; Aguirrezabalaga, 1984) to Senegal and Cape Verde islands (Núñez et al., 1999).

REGIONAL DISTRIBUTION:

G (Galician slope: Amourex, 1972)

I (Rasa de Zumaya: Campoy, 1982. Rasa de Algorri: Aguirrezabalaga, 1984)

J (Lanzarote: Núñez et al., 2005)

Notomastus formianus Eisig, 1887

Notomastus formianus Eisig, 1887. Fauna Flora Golf. Neapel, 16: 820. Type locality/origin: Gulf of Naples (Italy, Mediterranean Sea)

Global DistRIBUtion: It seems to be endemic in the Mediterranean Sea (Arvanitidis et al., 1999). Recorded in the Gulf of Naples (Eisig, 1887), Marseille (Harmelin, 1968), Gulf of Gaeta (Fauvel, 1927), Greece (Arvanitidis et al., 1999; Simboura \& Nicolaidou, 2001), Iberian Mediterranean Peninsula and Alboran Sea.

REGIONAL DISTRIBUTION:

A (Els Alfacs Bay: Capaccioni-Azzati, 1988)

B (Guardamar del Segura: Torres-Gavilá, 1989)

C (Chafarinas Islands: Torres-Gavilá, 2008)

Notomastus latericeus Sars 1851

Notomastus latericeus Sars, 1851. Nyt Mag. For Naturvid. 6: 199. Type locality/origin: Norway (NE Atlantic) 
Capitella rubicunda Keferstein 1862. Z. Wiss. Zool., 12: 123

Notomastus benedeni Claparède 1864. Mém. Soc. Phys. Hist. Nat. Genève, 17(2): 514

Arenia cruenta Quatrefages 1865. Hist. Nat. Annelés Mar. Eau Douce, 2: 250

Arenia fragilis Quatrefages 1865. Hist. Nat. Annelés Mar. Eau Douce, 2: 251

Sandanis rubicundus: Kinberg 1866. Öfvers. K. Vetensk. Akad. Förh, 22 (4): 343

Notomastus (Tremomastus) fertilis Eisig 1887. Fauna Flora Golf. Neapel, 16: 819

Notomastus (Tremomastus) rubicundus: Eisig 1887. Fauna Flora Golf. Neapel, 16: 863

Notomastus cruentus: Eisig 1887. Fauna Flora Golf. Neapel, 16: 865

Notomastus (Tremomastus) fragilis Eisig 1887. Fauna Flora Golf. Neapel, 16: 866

GlobAL Distribution: Cosmopolitan.

REGIONAL DISTRIBUTION: All sectors.

Notomastus lineatus Claparède, 1870

Notomastus lineatus Claparède, 1870. Mém. Soc. Phys. Hist. Nat. Genève, 20 (1): 18. Type locality/origin: Gulf of Naples (Italy, Mediterranean Sea)

? Notomastus sarsii Claparède, 1864. Mém. Soc. Phys. Hist. Nat. Genève, 17(2): 511

Notomastus (Clistomastus) lineatus Claparède: Eisig, 1887. Fauna Flora Golf. Neapel, 16: 810

GlOBAL DISTRIBUTION: Antarctic Ocean (Hartman 1969). Pacific from Canada to Mexico (Salazar-Vallejo \& Lodoño-Mesa, 2004) and Costa Rica (Dean, 2001). Western Atlantic from Panama and Gulf of Mexico (Ewing, 1984). Northeastern Atlantic. Mediterranean and Black Sea.

REGIONAL DISTRIBUTION:

A (Barcelona: Méndez \& Cardell, 1996; Els Alfacs Bay: Capaccioni, 1987)

B (Playa Levante, Benidorm: El Haddad, 2004)

D (Chafarinas Islands: López, 1995 as Notomastus cf. lineatus; Tena, 1996)

G (Vigo Ria: Ibáñez, 1973b; Viéitez, 1976, 1977, 1978. Pontevedra: Viéitez, 1978)

J (Tenerife Island: Pascual et al., 2000)

Notomastus profundus Eisig. 1887

Notomastus (Tremomastus) profundus Eisig, 1887. Fauna Flora Golf. Neapel, 16: 817. Type locality/origin: Gulf of Naples (Italy, Mediterranean Sea)

? Capitella major Claparède, 1870. Mém. Soc. Phys. Hist. Nat. Genève, 20 (1): 16

GlobAL Distribution: Eastern Atlantic from the British Isles (Hansson, 1998) to Morocco (Fauvel, 1936).
Mediterranean in Italy (Tyrrhenian, Ionian and Adriatic Seas) (Gravina \& Somaschini, 1990), Greece (Harmelin, 1969; Simboura \& Nicolaidou, 2001), Marmara Sea (Caspers, 1968) and Black Sea (in Rullier, 1963). Red Sea (Wehe \& Fiege, 2002).

REGIONAL DISTRIBUTION:

F (Algarve: Monteiro-Marqués, 1979. Setubal: Pinto, 1984)

G (Pontevedra: López-Jamar, 1978)

H (Continental shelf: Serrano et al., 2006)

REMARKS: This species is very close to Notomastus latericeus (Fauvel, 1936; Thomassin, 1970; Gambi \& Giangrande, 1986), so Thomassin (1970) proposed that these two species should be considered as synonymous. Some authors (e.g. Gambi \& Giangrande, 1986) used the name "Notomastus latericeus-profundus" in theirs lists to indicate the poor definition of taxonomic boundaries between these species.

Genus Peresiella Harmelin, 1968

Peresiella Harmelin, 1968. Rec. Trav. St. Mar. End., 43(59): 256

TYPE SPECIES: Peresiella clymenoides Harmelin, 1968

Peresiella clymenoides Harmelin, 1968

Peresiella clymenoides Harmelin, 1968. Rec. Trav. St. Mar. End., 43(59): 257. Type locality/origin: "Creta, Santorini, Marseille" (Mediterranean Sea).

GlobAL Distribution: Eastern Atlantic in Kinsale Port on the south coast of Ireland (Dinneen, 1982) and in Bay of Biscay (Martínez \& Adarraga, 2001). Japan Sea in Toyama Bay (Watanabe \& Shouzen, 1999). Mediterranean Sea.

REGIONAL DISTRIBUTION:

A (Rosas Bay: Desbruyères et al., 1972; Méndez \& Cardell, 1996. Els Alfacs Bay: Capaccioni, 1987; Martín et al., 2000; Brito et al., 2005)

B (Outer Valencia Port: Tena, 1992. Sierra Helada: Villarroya, 2005. Guardamar del Segura: Torres-Gavilá, 1989)

C (Cartagena Port: Malonda, 2009)

D (Chafarinas Islands: Torres-Gavilá, 2008)

I (Continental shelf of Guipúzcoa: Martínez \& Adarraga, 2001)

Genus Pseudocapitella Fauvel, 1913

Pseudocapitella Fauvel, 1913. Bull. Inst. Océanogr., 270: 79

TYPE SPECIES: Pseudocapitella incerta Fauvel 1913 
Pseudocapitella incerta Fauvel, 1913

Pseudocapitella incerta Fauvel, 1913. Bull. Inst. Océanogr., 270: 79. Type locality/origin: off Cannes (France, Mediterranean Sea)

GlobAl Distribution: Northeastern Atlantic in Cantabrian (Amoureux, 1973) and Galician (Amoureux, 1972) slopes. Mediterranean Sea.

REGIONAL DISTRIBUTION:

G (Galician slope: Amoureux, 1972)

H (Cantabrian slope: Amoureux, 1973)

Pseudocapitella incerta aberrans Amoureux, 1972

Pseudocapitella incerta aberrans Amoureux, 1972. Cah. Biol. Mar., 13: 19. Type locality/origin: off Galicia (Spain, NE Atlantic).

GLOBAL DISTRIBUTION: Known only from the type locality. 1972)

REGIONAL Distribution: G (Galician slope: Amoureux,

Genus Pseudoleiocapitella Harmelin, 1964

Pseudoleiocapitella Harmelin, 1964. Rec. Trav. St. Mar. End., 35(51): 90

TYPE SPECIES: Pseudoleiocapitella fauveli Harmelin, 1964

Pseudoleiocapitella fauveli Harmelin, 1964

Pseudoleiocapitella fauveli Harmelin, 1964. Rec. Trav. St. Mar. End., 35(51): 90. Type locality/origin: Marseille Bay (France, Mediterranean Sea).

GLOBAL Distribution: Mediterranean Sea with only one record outside of this area in the Gulf of Cádiz (López Serrano, 1999).

REGIONAL DISTRIBUTION:

A (Rosas Bay: Desbruyères et al., 1972. Blanes: Martín, 1986)

C (Almería: San Martín et al., 1990)

D (Tarifa: Sardá, 1984)

E (Mouth of Piedras River: López Serrano, 1999)

Genus Pseudomastus Capaccioni-Azzati \& Martín 1992

Pseudomastus Capaccioni-Azzati \& Martín 1992. Zool. Scri., 21(3): 247

TYPE SPECIES: Pseudomastus deltaicus Capaccioni-Azzati \& Martín, 1992

Pseudomastus deltaicus Capaccioni-Azzati \& Martín 1992 Pseudomastus deltaicus Capaccioni-Azzati \& Martín 1992. Zool. Scri., 247. Type locality/origin: Els Alfacs Bay (Spain, Mediterranean Sea)
Pseudoleiocapitella fauveli Harmelin: Capaccioni, 1987. Anél.

Poliqu. Ensenada Los Alfaques: 410 (non Harmelin, 1964)

Global Distribution: Mediterranean coast of Iberian Peninsula and Chafarinas Islands.

REGIONAL DISTRIBUTION:

A (Barcelona: Méndez \& Cardell, 1996)

B (Outer Valencia Port: Tena, 1992)

C (Cartagena Port: Malonda, 2009)

D (Chafarinas Islands: Torres-Gavilá, 2008)

\section{Acknowledgements}

We are grateful to Miguel Villena and Javier Sánchez Almazán who kindly provided the loan of Capitellidae specimens from the MNCN and to the staff of the "Servicio de Microscopía de la Universitat de Valencia" for help in SEM study. We would like to thank Vicky Zomot, Claudia García and Joan Soto for the careful revision of the English manuscript. We express our sincere thanks to Julio Parapar and Eduardo López for their critical reviews and comments which significantly improved this manuscript. This work was supported by the "Proyecto Fauna Ibérica CGL2004-04680-C10, Ministerio de Educación y Ciencia, Dirección General de Investigación".

\section{References}

Aguirrezabalaga, F., 1984. Contribución al estudio de los Anélidos Poliquetos de la Costa de Guipúzcoa. Munibe, 36: 119-130.

Aguirrezabalaga, F. \&. Carrera-Parra, L. F., 2006. Lumbrineridae (Polychaeta) from the Capbreton Canyon (Bay of Biskay, NE Atlantic) with the description of two new species. Scientia Marina, 70S3: 17-25. doi: 10.3989/scimar.2006.70s317

Aguirrezabalaga, F. \& Ceberio, A., 2003. Dorvilleidae (Polychaeta) from the Capbreton Canyon Bay of Biscay, NE Atlantic) with the description of Pettiboneia sanmartini sp. nov. Cahiers de Biologie Marine, 44: 41-48.

Aguirrezabalaga, F. \& Ceberio, A., 2005. Spionidae (Annelida: Polychaeta) from the Capbreton Canyon (Bay of Biskay, NE Atlantic) with descriptions of a new genus and three new species. Marine Biology Research, 1: 267-280. doi: 10.1080/17451000500262066

Aguirrezabalaga, F. \& Ceberio, A., 2006. Flabelligena gasgognensis sp. nov. (Polychaeta: Acrocirridae), a new species from the Capbreton Canyon (Bay of Biskay, NE Atlantic). Oceanography of the Bay of Biskay. Scientia Marina, 70S1: 141-147.

Aguirrezabalaga, F., Ceberio, A. \& Fiege, D., 2001. Octomagelona bizkaiensis (Polychaeta: Magelonidae) a new genus and species from the Capbreton Canyon 
(Bay of Biscay, north-east Atlantic). Journal of the Marine Biological Association of the United Kingdom, 81: 221-22. doi: 10.1017/S0025315401003678

Aguirrezabalaga, F., Ceberio, A. \& Paxton, H., 2002. Onuphidae (Polychaeta) from the Capbreton (Bay of Biskay, NE Atlantic), with the description of Paradiopatra capbretonensis sp. nov. Steenstrupia, 27(1): 19-28.

Aguirrezabalaga, F., Ibáñez, M. \& Ros, J., 1986. Taxocenosis anelidianas en el piso circalitoral de la costa vasca. Actas $V$ Simposio Ibérico de Estudios del Bentos Marino, 2: 149-159.

Acero, M. L. \& San Martín, G., 1986. Poliquetos epibiontes del primer horizonte de algas fotófilas en las provincias de Cádiz y Málaga. Estudio faunístico comparado. Boletín de la Real Sociedad Española de Historia Natural (Sección Biológica), 82(1-4): 5-24.

Alós, C., 1988. Anélidos Poliquetos del Cabo de Creus (Alt Ampordá). Tesis Doctoral. Universidad de Barcelona. Barcelona. 838 pp. (inédita).

Alós, C. \& Pereira, F., 1989. Estudio de la población de Anélidos Poliquetos de Posidonia oceanica. Posidonia Newsletter, 2(1): 5-16.

Amaral, A. C., 1980. Breve caracterização dos gêneros da familia Capitellidae Grube (Annelida, Polychaeta) e descrição de Nonatus longilneus gen. sp. nov. Bulletin of the Institute of Oceanography, São Paulo, 29(1): 99-106.

Amoureux, L., 1972. Annélides Polychètes recueillies sur les pentes du talus continental, au large de la Galice (Espagne). Campagnes 1967 et 1968 de la «Thalassa». Cahiers de Biologie Marine, 13: 63-89.

Amoureux, L., 1973. Annélides Polychètes recueillies sur les pentes du talus continental au nord de la côte espagnole. Campagne 1970 de la «Thalassa». Cahiers de Biologie Marine, 14: 429-452.

Amoureux , L., 1974. Annélides Polychetes du banc Le Danois. Campagne 1971 de la «Thalassa » (octobre). Boletín de la Real Sociedad Española de Historia Natural (Sección Biológica), 72: 101-127.

Amoureux, L., 1983a. Annélides polychètes du Golfe d'Aqaba (Mer Rouge). Description d'un genre nouveau et de deux espèces nouvelles. Bulletin $d u$ Muséum National d'Histoire Naturelle, 4(5): 723-742.

Amoureux, L., 1983b. Annélides polychètes de Mer Adriatique et de Mer Rouge. Nouvelles observations, Rapports et procès-verbaux des réunions. Commission Internationale pour l'exploration scientifique de la Mer Méditerranée Monaco, 28(3): 253-254.

Ariño, A., 1987. Bibliografía ibérica de poliquetos. Base de datos y catálogo de especies. Publicaciones de Biología de la Universidad de Navarra, serie Zoológica, 16. EUNSA. Pamplona. 172 pp.

Arvanitidis, C., 2000. Polychaete fauna of the Aegean Sea: Inventory and new information. Bulletin of Marine Science, 66(1): 73-96.
Arvanitidis, C., Koutsoubas, D., Dounas, C. \& Eleftheriou, A., 1999. Annelid fauna of a Mediterranean lagoon (Gialova Lagoon, South-West Greece): Community structure in a severely fluctuating environment. Journal of the Marine Biological Association of the United Kingdom, 79: 849-856.

Augener, H., 1914. Polychaeta II. Sedentaria. In: W. Michaelsen \& R. Hartmeyer (eds.). Die Fauna Südwest-Australiens. Vol. 5. Gustav Fischer. Jena. $170 \mathrm{pp}$.

Augener, H., 1918. Polychaeta. In: W. Michaelsen (ed.). Beiträgen zur Kenntnis der Meeresfauna Westafrikas. Vol. 2. Friedrichsen. Hamburg: 67-625.

Bellan, G., 1959. Campagnes de la Calypso: Mer d'Alboran. 2. Annélides Polychètes. Annales de l'Institut Océanographique, 37: 315-342.

Bellan, G., 1964. Contribution à l'étude systématique bionomique et écologique des Annélides Polychètes de la Méditerranée. Recueil des Travaux de la Station Marine d'Endoume, 49(33): 1-372.

Beneden, P. J. van, 1857. Histoire naturelle du genre Capitella de Blainville ou du Lumbriconais d'Oersted, comprenant la structure anatomique, le développement et les caractères extérieures. Bulletin de l'Académie Royale Belgique, Bruxelles, 3: 137-162.

Ben-Eliahu, M. N., 1976. Polychaete cryptofauna from rims of similar intertidal Vermetid reefs on the Mediterranean coast of Israel and in the Gulf of Elat. Sedentaria. Israel Journal Zoology, 25: 121-155.

Ben-Eliahu, M. N. \& Fiege, D., 1995. Polychaeta from the continental shelf and slope of Israel collected by the 'Meteor' 5 expedition (1987). Senckenbergiana. Maritime, 25(4/6): 85-105.

Blainville, H. de, 1828. Dictionnaire des Sciences Naturelles, dans lequel on traite méthodiquement des différens êtres de la nature, considérés soit en euxmêmes, d'après l'état actuel de nos connaisance, soit relativement à l'utilité qu'en peuvent retirer la médicine, l'agriculture, le commerce et les arts. Suivi d'une biographie des plus célèbres naturalistes. Vol. LVII. Levrault. Strasbourg. 628 pp.

Blake, J. A., 2000. Family Capitellidae Grube, 1862. In: J. A. Blake, B. Hilbig, \& P. H. Scott, (eds.). Taxonomic atlas of the benthic fauna of the Santa Maria Basin and the western Santa Barbara Channel. Vol. 7. The Annelida Part 4, Polychaeta: Flabelligeridae to Ampharetidae. Santa Barbara Muséum of Natural History. Santa Barbara: 47-96.

Blake, J. A., 2009. Redescription of Capitella capitata (Fabricius) from West Greenland and designation of a neotype (Polychaeta, Capitellidae). Zoosymposia, 2: $55-80$.

Brito, M. C., 1999. Estudio de las comunidades intersticiales del sebadal (Cymodocea nodosa) en Canarias. Tesis Doctoral. Universidad de La Laguna. Santa Cruz de Tenerife. 610 pp. (inédita). 
Brito, M. C., Martín, D. \& Núñez, J., 2005. Polychaetes associated to a Cymodocea nodosa meadow in the Canary Islands: assemblage structure, temporal variability and vertical distribution compared to other Mediterranean seagrass meadows. Marine Biology, 146: 467-481. doi: 10.1007/s00227-004-1460-1

Cacabelos, E., 2005 Cartografía bionómica de las poblaciones macrobentónicas de sustratos blandos de la Ensenada de San Simón (Galicia). Tesis Doctoral. Universidad de Vigo. Vigo. (inédita).

Cacabelos, E., Gestoso, I. \& Troncoso, J., 2008a. Macrobenthic fauna in the Ensenada de San Simón (Galicia, north-west Spain). Journal of the Marine Biological Association of the United Kingdom, 88(2): 237-245. doi: 10.1017/S0025315408000660

Cacabelos, E., Moreira, J. \& Troncoso, J., 2008 b. Distribution of Polychaeta in soft-bottoms of a Galician Ria (NW Spain). Scientia Marina, 72(4): 655-667. doi: 10.3989/scimar.2008.72n4655

Camp, J., 1976. Comunidades bentónicas de sustrato duro del litoral NE español. IV. Poliquetos. Investigación Pesquera, 40(2): 533-550.

Campoy, A., 1979. Lista de especies de Anélidos Poliquetos conocidas de las costas de la Península Ibérica. Investigación Pesquera, 43(3): 737-766.

Campoy, A., 1982. Fauna de España. Fauna de Anélidos Poliquetos de la Península Ibérica. Ediciones de la Universidad de Navarra. Pamplona. 780 pp.

Campoy, A. \& Jordana, R., 1978. Contribución al conocimiento de la fauna de Anélidos Poliquetos de las costas españolas: Nota sobre una relación de especies capturadas en Blanes (Gerona) y Aguilas (Murcia). Boletín de la Real Sociedad Española de Historia Natural, 76(1-2): 49-56.

Capaccioni, R., 1983. Anélidos poliquetos del Mar Menor: faunistica y ecología. Tesis de Licenciatura. Universidad de Valencia. Valencia. 205 pp. (inédita).

Capaccioni, R., 1987. Anélidos Poliquetos de la Ensenada de los Alfaques (Delta del Ebro, Mediterráneo Occidental). Tesis Doctoral. Universidad de Valencia, Valencia. 533 pp. (inédita).

Capaccioni-Azzati, R., 1988. Prionospio multibranchiata (Polychaeta, Spionidae), Notomastus aberans y $N$. formianus (Polychaeta, Capitellidae) en el litoral de la Península Ibérica. Miscel-lania Zooogica, 12: 47-56.

Capaccioni-Azzati, R. \& Martín, D., 1992. Pseudomastus deltaicus, gen. et sp.n. (Polychaeta: Capitellidae) from a shallow-water bay in the North-Western Mediterranean Sea. Zoologica Scripta, 21(3): 247- 250. doi: 10.1111/j. 1463-6409.1992.tb00328.x

Capaccioni-Azzati, R., Torres-Gavilá, F. J. \& Tena, J., 1992. Dos nuevos géneros de anélidos poliquetos para la península ibérica: Neopseudocapitella Rullier and Amoureux, 1979 y Demonax Kinberg, 1867. Thalassas, 10: 115-121.
Capaccioni Azzati, R., 1985. Sobre la presencia de Mediomastus fragilis Rasmussen, 1973 (Polychaeta, Capitellidae). Investigación Pesquera, 49(1): 47-53.

Carvalho, S., Ravara, A., Quintito, V. \& Rodrigues, A. M., 2001. Macrobenthic community characterisation of an estuary from the western coast of Portugal (Sado estuary) prior to dredging operations. Boletin del Instituto Español de Oceanografia, 17(1 y 2): 179-180.

Carpine, C., 1970. Écologie de l'étage bathyal dans la Méditerranée occidentale. Mémoires de l'Institut Océanographique, Monaco, 2: 1-146.

Caspers, H., 1968. La macrofaune benthique du Bosphore et les problèmes de l'infiltration des éléments méditerranéens dans la mer Noire. Rapport et procès verbaux des réunions. Commission internationale pour l'exploration scientifique de la Mer Méditerranée, 19(2) :107-115.

Castelli, A., Abbiati, M., Badalamenti, F., Bianchi, C. N., Cantone, G., Gambi, M. C., Giangrande, A., Gravina, M. F., Lanera, P., Lardicci, C., Somaschini, A. \& Sordino, P., 1995. Annelida Polychaeta, Pogonophora, Echiura, Sipuncula. In: A. Minelli, S. Ruffo, \& S. La Posta (eds.). Checklist delle specie della fauna italiana. Calderini. Bologna: 1-45.

Claparède, E., 1864. Glanures Zootomiques parmi les Annélides de Port-Vendres (Pyrénées Orientales). Mémoires de la Société de Physique et d'Histoire Naturelle de Genève, 17(2): 463-600.

Claparède, E., 1870. Les Annélides chétopodes du Golfe de Naples. Supplément. Mémoires de la Société de Physique et d'Histoire Naturelle de Genève, 20: 1178.

Cunha, M. R. \& Ravara, A., 2003. Macrofaunal zonation and sediment properties on a low-energy, mesotidal sandy beach (Ria de Aveiro) (northwestern Portugal). Boletín del Instituto Español de Oceanografia, 19(14): 235-246.

Day, J. H., 1957. The Polychaeta of South Africa. Part 4: New species from Natal and Moçambique. Annals of the Natal Museum, 14(1): 59-129.

Day, J. H., 1961. The polychaet fauna of South Africa. Part 6. Sedentary species dredged off Cape coasts with a few records from the shore. Journal of the Linnean Society of London, 44 (299): 463-560. doi: 10.1111/j.1096-3642.1961.tb01623.x

Day, J. H., 1967. A Monograph on the Polychaeta of Southern Africa. British Muséum (Natural History) Publication 656. London. 2 vols: Pt 1, Errantia, 458 pp., Pt 2, Sedentaria, 459-878.

Day, J. H., 1973. New Polychaeta from Beaufort, with a key to all species recorded from North Carolina. National Marine Fisheries Service Circular, 375: 1140.

Dean, H. K., 2001. Capitellidae (Annelida: Polychaeta) from the Pacific coast of Costa Rica. Revista de Biología Tropical, 49(Suppl. 2): 69-84. 
Desbruyères, D., Guille, A. \& Ramos, J., 1972. Bionomie benthique du plateau continental de la côte catalane espagnole. Vie et Milieu, 23(2B): 335-363.

Dinneen, P., 1982. Peresiella clymenoides Harmelin, 1968; a capitellid polychaete new to Ireland and Great Britain. Irish Naturalists'Journal, 20(11): 471-475.

Eisig, H., 1887. Monographie der Capitelliden des Golfes von Neapel. Fauna und Flora des Golfes von Neapel, 16: 1-906.

El Haddad, M., 2004. Contribución al estudio de la macrofauna bentónica de las playas Levante y Poniente de Benidorm. Trabajo de Investigación (DEA). Universidad de Valencia. Valencia. 57 pp. (inédita).

El Haddad, M., Assadi, C., Tasso, V., Villarroya, I., Gallardo, F. J., Capaccioni Azzati, R., García Carrascosa, M., Sáez, J. \& Monforte, F., 2007. Catálogo preliminar de especies no indígenas de la biota marina del Puerto de Valencia (Mediterráneo Occidental) y su potencial invasivo. Actas $I I^{\circ}$ Congreso Nacional sobre especies exóticas invasoras "EEI 2006”. "EEI 2006”. GEIB, Serie Técnica: 202-224.

Ewing, R. M., 1982. A partial revision of the genus Notomastus (Polychaeta: Capitellidae) with a description of a new species from the Gulf of Mexico. Proceedings of the Biological Society of Washington, 95: 232-237.

Ewing, R. M., 1984. Family Capitellidae Grube, 1862. In: J.M. Uebelacker \& P.G. Johnson (eds.). Taxonomic guide to the Polychaetes of the Northern Gulf of Mexico. Vol. 2: 14.1 to 14-47. Barry A. Vittor \& Associates. Mobile, Inc. Alabama. 47 pp.

Fabricius, O., 1780. Fauna Groenlandica. Hafniae et Lipsiae. 452 pp.

Fauvel, P., 1913. Quatrième note préliminaire sur les polychètes provenant des campagnes de 1" "Hirondelle" et de la "Princesse-Alice", ou déposées dans le Musée Océanographique de Monaco. Bulletin de l'Institut Océanographique, Monaco, 270: 1-80.

Fauvel, P., 1914. Annélides Polychètes non-pélagiques provenant des campagnes de 1" Hirondelle" et de la "Princesse-Alice" (1885-1910). Résultats des Campagnes Scientifiques du Prince Albert $1^{\circ}$ de Monaco, 46: 1-432.

Fauvel, P., 1927. Polychètes sédentaires. Addenda aux errantes, Archiannélides, Myzostomaires. In: Faune de France, vol. XVI. Le Chevalier. Paris. 494 pp.

Fauvel, P., 1936. Contribution à la faune des Annélides Polychètes du Maroc. Mémoires de la Société des Sciences Naturelles du Maroc, 43: 1-143.

Fredette, T. J., 1982. Evidence of ontogenetic setal changes in Heteromastus filiformis (Polychaeta: Capitellidae). Proceedings of the Biological Society of Washington, 95: 194-197.

Gambi, M. C. \& Giangrande, A., 1986. Distribution of soft-bottom Polychetes in two coastal areas of the
Tyrrhenian Sea (Italy): structural analysis. Estuarine Coastal and Shelf Science, 23: 847-862. doi: 10.1016/ 0272-7714(86)90076-4

García, O. L., García, M. A., Mora, J. \& Rodríguez, C., 1979. Adiciones al catalogo de los Anélidos poliquetos de España. Trabajos Compostelanos de Biología, 8: 55-60.

García Alvarez, O. L., Míguez Rodríguez, L., Fernández Abelleira, J., Ortiz Delgado, S. \& Veloso Ríos, M., 1993. Poblamientos faunísticos intermareales de sustrato duro en la ría de La Coruña. Publicaciones especiales del Instituto español de Oceanografía, 11: 267-274.

Garmendia, L., Marquiegui, M., Aguirrezabalaga, F., Cruz, I. \& Cantón, L., 2003. Efecto de la desaparición de los vertidos de aguas residuales urbanas sobre la comunidad reducida de Macoma en las islas del estuario del río Bidasoa (golfo de Vizcaya). Boletín del Instituto Español de Oceanografia, 19(1-4): 265-281.

Giangrande, A. \& Gambi, M. C., 1986. Polychètes d'une pelouse a Cymodocea nodosa (Ucria) Aschers. du Golfe de Salerno (Mer Tyrrhénienne). Vie et Milieu, 36(3): 185-190.

Glemarec, M., 1969. Les peuplements benthiques du plateau continental Nord-Gascogne. Tesis Doctoral. Universidad de Brest. 167 pp. (inédita).

Gravier, C., 1901. Sur un Capitellien d'eau douce (Eisigella n.g., ouanaryensis n. sp.). Bulletin du Muséum d'Histoire Naturelle, 7: 402-404.

Gravier, C., 1911. Expédition antarctique francaise du 'Pourquoi-Pas' dirigée par le Dr J. B. Charcot (19081910). Espèces nouvelles d'annélides polychetes. Bulletin du Muséum National d'Histoire Naturelle, 17: 310-316.

Gravina, M. F. \& Somaschini, A., 1988. New record of Mediomastus fragilis Rasmussen, 1973 for the Italian Fauna and notes on the genus Mediomastus Hartman, 1944 (Polychaeta, Capitellidae). Atti della Società Toscana di Scienze Naturali, Memorie (Serie B), 95: 59-67.

Gravina, M. F. \& Somaschini, A., 1990. Censimento dei Policheti dei Mari italiani: Capitellidae Grube, 1862. Atti della Società Toscana di Scienze Naturali, Memorie (Serie B), 97: 259-285.

Green, K. D., 2002. Capitellidae (Polychaeta) from the Andaman Sea. In: D. Eibye-Jacobsen, (ed.). Proceedings of the International Workshop on the Polychaetes of the Andaman Sea. Department of Fisheries. Thailand, 24: 249-343.

Grube, A. E., 1846. Beschreibung neuer oder wenig bekannter Anneliden. Zweiter Beitrag: Corephorus elegans Gr., Ammochares ottonis Gr., Dasymallus caducus Gr., Scalis minax Gr. Archiv für Naturgeschichte, 12(1): 161-171.

Grube, A. E., 1850. Die Familien der Anneliden. Archiv für Naturgeschichte, 16(1): 249-364. 
Grube, A. E., 1862. Noch ein Word ubre die Capitellen und ihre Stellung im Systeme der Anneliden. Archiv für Naturgeschichte, 28: 366-378.

Grube, A. E., 1867. Anneliden. Reise der Österreichischen Fregatte Novara um die Erde in den Jahren 1857, 1858, 1859. Unter den Befehlen des Commodore B. von Wüllerstorf-Urbair. Zoolgischer Theil, 2(3): 1-46.

Grube, A. E., 1878. Annulata Semperiana. Beiträge zur Kenntnis der Annelidenfauna der Philippinen nach den von Herrn Prof. Semper mitgebrachten Sammlungen. Mémoires de l'Académie Impériale des Sciences de St. Pétersbourg, 7ème série, 25: 1-300.

Hansson, H. G., 1998. NEAT (North East Atlantic Taxa): South Scandinavian marine Annelida Check-List. Internet PDF Edition, August 1998. [http://www. tmbl.gu.se].

Harmelin, J. G., 1964. Étude de l'endofaune des «mattes» d'herbiers de Posidonia oceanica Delile. Recueil des Travaux de la Station Marine d'Endoume, 35(51): 43105.

Harmelin, J. G., 1968. Note sur trois Capitellidae (Annélides polychètes) récoltés en Mediterranée, avec description d'un nouveau genre: Peresiella. Recueil des Travaux de la Station Marine d'Endoume, 59: 253-259.

Harmelin, J. G., 1969. Contribution a l'étude de l'endofaune des prairies d'Halophila stipulacea de Méditerranée Orientale. I. Annélides Polychètes. Recueil des Travaux de la Station Marine d'Endoume, 45: 305-316.

Hartman, O., 1944. Polychaetous annelids from California, including the descriptions of two new genera and nine new species. Allan Hancock Pacific Expeditions, 10(2): 239-307.

Hartman, O., 1947. Polychaetous annelids. Part VII. Capitellidae. Allan Hancock Pacific Expeditions, 10(4): 391-481.

Hartman, O., 1969. Atlas of Sedentariate Polychaetous Annelids from California. Allan Hancock Foundation, University of Southern California. Los Angeles. $812 \mathrm{pp}$.

Hartmann-Schröder, G., 1971. Annelida, Borstenwürmer, Polychaeta. In: Die Tierwelt Deutschlands und der angrenzenden Meersteile nach ihrenMerkmalen und nachihrer Lebensweise, 58. Gustav Fischer Verlag. Jena. $594 \mathrm{pp}$.

Hartmann-Schröder, G., 1974. Zur Kenntnis des Eulitorals der afrikanischen Westküste zwischen Angola und Kap der Gunten Hoffnung und der afrikanischen Ostküste von Südafrika und Mocambique unter besonderer Berücksichtigung der Polychaeten und Ostracoden. Teil II. Die Polychaeten des Untersuchungsgebietes. Mitteilungen aus dem Hamburgischen Zoologischen Muséum und Institut, 187: 95-228.

Hernández-Alcántara， P. \& Solís-Weiss, V., 1998. Capitellids (Polychaeta: Capitellidae) from the conti- nental shelf of the Gulf of California, with the description of a new species, Notomastus angelicae. Proceedings of the Biological Society of Washington, 111(3): 708-719.

Hutchings, P. A., 2000. Family Capitellidae. In: P. L. Beesley, G. J. B. Ross \& C. J. Glasby eds.). Polychaetes and Allies: The Southern Synthesis. Fauna of Australia. Vol.4A. Polychaeta, Myzostomida, Pogonophora, Echiura, Sipuncula. CSIRO Publishing: Melbourne: 67-72.

Hutchings, P. A. \& Rainer, S., 1981. Designation of a neotype of Capitella filiformis Claparède, 1864, type species of the genus Heteromastus (Polychaeta: Capitellidae). Records of the Australian Museum, 34: 373-380.

Ibáñez, M., 1973a. Catálogo de los anélidos poliquetos citados en las costas españolas. Cuadernos de Ciencias Biológicas, 2(2): 121-140,

Ibáñez, M., 1973b. Contribución al estudio ecológico de los anélidos poliquetos de la Península Ibérica. Tesis Doctoral. Universidad de Complutense de Madrid, Madrid. 125 pp. (inédita).

Ibáñez, M., Romero, A., San Vicente, C. \& Zaballa, K., 1984. La contaminación marina en Gipuzkoa II. Estudio de los indicadores biológicos de la contaminación en las rías del Urola, Oria y Urumea. Lurralde, 7: 197-216.

Johnston, G., 1827. Contributions to the British Fauna. Zoological Journal London, 3: 328-336.

Johnston, G., 1865. A catalogue of the British non-parasitical worms in the collection of the British Museum. London. 365 pp.

Keferstein, W., 1862. Untersuchungen über niedere Seethiere. Zeitschift für Wissenschaftliche Zoologie, 12(1): 1-147.

Kinberg, J. G. H., 1866. Annulata Nova, Öfversigt af Kongliga Vetenskaps-Akademiens Förhandlingar, 22(4): 239-258.

Kinberg, J. G. H., 1867. Annulata Nova. Öfversigt af Kongliga Vetenskaps-Akademiens Förbandlingar, 23: 337-357.

Kirkegaard, J. B., 1959. The Polychaeta of West Africa Part I. Sedentary species. Atlantide Report, 5: 7-117.

La Greca, M., 1949. Notes sur les Polychètes du Bosphore. Istanbul Üniversitesi Fen Fakültesi Mecmuasi, Cilt XIV, Serie B, 3: 153-169.

Lanera, R. \& Gambi, M. C., 1993. Polychaete distribution in some Cymodocea nodosa meadows around the island of Ischia (Gulf of Naples, Italy). Oebalia, 19: 89-104.

Langerhans, P., 1880. Die Wurmfauna von Madeira III. Zeitschrift für Wissenschaftliche Zoologie, 34: 87143.

Langerhans, P., 1881. Ueber einige canarische Anneliden. Nova Acta Leopoldina, 42: 93-124. 
Lastra, M., 1991. Cartografía y dinámica de la macrofauna bentónica submareal de los sustratos blandos de la Bahía de Santander. Tesis Doctoral. Universidad de Santiago de Compostela. 383 pp (inédita).

López, E., 1995. Anélidos Poliquetos de sustratos duros de las Islas Chafarinas. Tesis Doctoral. Universidad Autónoma de Madrid, Madrid. 672 pp. (inédita).

López-Cotelo, I., Viéitez, J. M. \& Díaz-Pineda, F., 1982. Tipos de comunidades bentónicas de la playa del Puntal (bahía de Santander). Cahiers de Biologie Marine, 23: 53-69.

López-Jamar, E., 1978. Primeros datos sobre la biomasa y la composición del bentos infaunal de la Ría de Pontevedra, en relación con el contenido de la materia orgánica del sedimento. Boletín del Instituto Español de Oceanografia, 4: 57-69.

López-Jamar, E., 1981. Spatial distribution of the infaunal benthic communities of the Ría de Muros, NW Spain. Marine Biology, 63: 29-37.

López-Jamar, E., 1982. Distribución espacial de las comunidades bentónicas infaunales de la Ría de Arosa. Boletín del Instituto Español de Oceanógrafa, 7(2): 255-268.

López Serrano, L., 1999. Estudio de la macrofauna bentónica de la desembocadura del río Piedras (Huelva). Tesis Doctoral. Universidad Complutense de Madrid, Madrid. 240 pp. (inédita).

Louridom, E, Cacabelos, E. \& Troncoso, J., 2008. Patterns of distribution of the polychaete fauna in subtidal soft sediments of the Ría de Aldán (northwestern Spain). Journal of the Marine Biological Association of the United Kingdom, 88(2): 263-275.

Malonda, I., 2009. Estudio de la taxocenosis de Poliquetos como parte de la evaluación de la calidad ambiental en el puerto de Cartagena (Murcia, España). Tesis Doctoral. Universidad Autónoma de Madrid, Madrid. 320 pp (inédita).

Martín, D., 1986. Anélidos Poliquetos y Moluscos asociados a algas calcáreas. Tesis de Licenciatura. Universidad de Barcelona. Barcelona. 261 pp. (inédita).

Martín, D., Ballesteros, E., Gili, J. M. \& Palacín, C., 1993. Small scale structure of infaunal polychaete communities in an estuarine environment: Methodological approach. Coastal and Shelf Science, 36: 47-58. http://dx.doi.org/10.1006/ecss. 1993.1004

Martín, D., Pinedo, S. \& Sardá, R., 2000. Distribution patterns and trophic structure of soft-bottom polychaete assemblages in a north-western Mediterranean shallow-water bay. Ophelia, 53(1): 1-17.

Martínez, J. \& Adarraga, L., 2001. Distribución batimétrica de comunidades macrobentónicas de sustrato blando en la plataforma continental de Guipuzcoa (golfo de Vizcaya). Boletín de la Real Sociedad Española de Historia Natural (Sección Biológica), 17(1-2): 33-48.
Martínez, J., Adarraga, L. \& Ruiz, J. M., 2005. Proyecto Fauna de los invertebrados marinos de la costa vasca: EUSKALBENTOS I. 1. Cartografía bionómica de los fondos blandos naturales de la costa de Gipuzkoa: Caracterización de las comunidades, inventarios de especies, censos y establecimiento de bioindicadores de calidad ambiental. Informe inédito. Departamento de Medio Ambiente y Ordenación del Territorio, Gobierno Vasco. Vitoria-Gasteiz.

Martínez, J., Adarraga, L. \& Ruiz, J. M., 2006. Proyecto Fauna de los invertebrados marinos de la costa vasca: EUSKALBENTOS II. 2. Cartografía bionómica de los fondos blandos naturales de la costa de Bizkaia: Caracterización de las comunidades, inventarios de especies, censos y establecimiento de bioindicadores de calidadambiental. Informe inédito. Departamento de Medio Ambiente y Ordenación del Territorio, Gobierno Vasco. Vitoria-Gasteiz.

Martínez, J., Adarraga, L. \& Ruiz, J. M., 2007a. Proyecto Fauna de los invertebrados marinos de la costa vasca: EUSKALBENTOS III. 3. Cartografía bionómica de los fondos del margen superior del Talud Continental de la costa vasca: Caracterización de las comunidades, inventarios de especies, censos y establecimiento de bioindicadores de calidad ambiental. Informe inédito. Departamento de Medio Ambiente y Ordenación del Territorio, Gobierno Vasco. Vitoria-Gasteiz.

Martínez, J., Adarraga, L. \& Ruiz, J. M., $2007 \mathrm{~b}$. Tipificación de poblaciones bentónicas de los fondos blandos de la plataforma continental de Guipúzcoa (sureste del golfo de Vizcaya). Boletín del Instituto Español de Oceanografía, 23(1-4): 85-110.

McCammon, J. A. \& Stull, J. K., 1978. A new genus and species of Capitellidae (Polychaeta) from California. Bulletin of the Southern California Academy of Sciences, 77(1): 40-43.

McIntosh, W. C., 1885. Report on the Annelida Polychaeta collected by H. M. S. Challenger during the years 1873-76. Report on the Scientific Results of the voyage of H.M.S. Challenger (Zoology), 12: 1-554.

Méndez, N., 2006. Life cycle of Capitella sp. Y (Polychaeta: Capitellidae) from Estero del Yugo, Mazatlán, Mexico. Journal of the Marine Biological Association of the United Kingdom, 86: 263-269. doi: http://dx.doi.org/10.1017/S0025315406013117

Méndez, N. \& Cardell, M. J., 1996. Littoral annelid polychaetes inhabiting soft bottoms of the Barcelona (Catalonia, N.E. Spain). Miscel-lania Zoologica, 19(1): 119-147.

Méndez, N., Linke-Gamenick, I. \& Forbes, V. E., 2000. Variability in reproductive mode and larval development within the Capitella capitata species-complex. Invertebrate Reproduction and Development, 38: 131-142. doi: 10.1080/07924259.2000.9652448

Mesnil, F., 1897. Note sur un Capitellien nouveau (Capitellides n. gen., Giardi n. sp.). Zoologischer Anzeiger, 20: 441-443. 
Monteiro-Marqués, V., 1979. Contribution à l'étude du plateau continental des côtes portugaises (Algarve, Sud Portugal): étude des peuplements de substrats meubles. Tethys, 9(2): 191-195.

Moreira, J., 2003. La fauna bentónica de la Ensenada de Baiona (Galicia NO península Ibérica): diversidad, análisis de las comunidades, dinámica de poblaciones $y$ distribución vertical. Tesis Doctoral. Universidad de Vigo. (inédita).

Moreira J., Domínguez M. \& Troncoso, J., 2003. Distribución vertical de la macrofauna submareal en la Ensenada de Baiona (Galicia, N.O. España). Nova Acta Científica Compostelana (Bioloxía), 13: 89-102.

Moreira J., Quintas P. \& Troncoso J., 2006. Spatial distribution of soft-bottom polychaete annelids in the Ensenada de Baiona (Ría de Vigo, Galicia, northwest Spain). Scientia Marina, 70S3: 217-224.

Nardo, G. D., 1847. Prospetto della fauna marine volgare del Veneto estuario con cenni sulle principali specie commestibili dell'Adriatico, ecc. In: G. Antonelli (ed.). Venezia e le sue lagune. Giorgio Franz in Monaco: 1-45.

Nogueira de Carvalho, R., 1929. Catalogo de colecçao de Invertebrados de Portugal existentes no Museu Zoológico da Universidade de Coimbra. Vermes. I. Polychaeta. Memórias e Estudos do Museu Zoológico da Universidade de Coimbra, 37: 1-16.

Núñez, J., Brito, M. C. \& Docoito, J. R., 2005. Anélidos Poliquetos de Canarias: Catálogo de especies, distribución y hábitats. Vieraea, 33: 297-321.

Núñez, J., Viera, G., Riera, R. \& Brito, M. C., 1999. Anélidos Poliquetos bentónicos de las Islas de Cabo Verde: primer catálogo faunístico. Revista de la Academia Canaria de Ciencias, 11(3-4): 135-172.

Oersted, A. S., 1842. Conspectus generum specierumque Naidum ad faunam Danicam pertinentium. Naturhistorisk Tidsskrift, 4: 128-140.

Parapar, J., 1991. Anélidos Poliquetos bentónicos de la Ría de Ferrol (Galicia). Tesis Doctoral. Universidad de Santiago de Compostela. Santiago de Compostela. 1104 pp. (inédita).

Parapar, J., Besteiro, C., \& Urgorri, V., 1996. Inventario dos Poliquetos de Galicia (Annelida: Polychaeta). Cadernos da Área de Ciencias Biolóxicas (Inventarios), XVI. Publicacións do Seminario de Estudos Galegos. 178 pp.

Parapar, J., Martínez-Ansemil, E., Caramelo, C., Collado, R. \& Schmeltz, R., 2009. Polychaetes and oligochaetes associated with intertidal rocky shores in a semi-enclosed industrial and urban embayment, with the description of two new species. Helgoland Marine Research, 63: 293-308.

Parapar, J. \& Moreira, J., 2009. Polychaeta of the 'DIVA-Artabria I' project (cruise 2002) in the continental shelf and upper slope off Galicia (NW Spain). Cahiers de Biologie Marine, 50: 57-78.
Parapar, J., San Martín, G., Urgorri, V. \& Besteiro, C., 1993. Anélidos poliquetos mesopsámmicos sublitorales de la costa de Ceuta (España). Cahiers de Biologie Marine, 34: 363-381.

Pascual, M., Núñez, J., Brito, M.C. \& Riera, R., 2000. Escolécidos (Polychaeta: Scolecida) endobiontes de esponjas de Canarias y Madeira. Revista de la Academia Canaria de Ciencias, 12(3-4): 55-65.

Pinto, P., 1984. Nota preliminar sobre a detecção da heterogeneidade do padrão de distribução espacial em povoamentos bentónicos. In: M. Monteiro Marques (ed.). Actas IV Simposio Ibérico de Estudios del Bentos Marino, 1: 1-9.

Planas, M., 1986. Dinámica de las poblaciones de la macrofauna bentónica intermareal de la ensenada de Lourizán - Ría de Pontevedra. Tesis Doctoral. Universidad de Santiago de Compostela, Santiago de Compostela. 533 pp. (inédita).

Quatrefages, A. de, 1865. Histoire Naturelle des Annelés marins et d'eau douce. Annélides et Géphyriens. Vol. 2. Librarie Encyclopédique de Roret. Paris. 336 pp.

Quintas, P., 2005. Distribución espacial y temporal de los moluscos y anélidos poliquetos asociados a las praderas de Zostera marina L. y Zostera noltii Hornem. En la Ensenada de O Grove (Galicia, España). Tesis Doctoral (inédita). Universidad de Vigo. Vigo. (inédita).

Ramírez-Llodra, E., Ballesteros, M., Company, J. B., Dantart, L. \& Sardá, F., 2008. Spatio-temporal variations of biomass and abundance in bathyal non-crustacean megafauna in the Catalan Sea (North-western Mediterranean). Marine Biology, 153: 297-309. doi: 10.1007/s00227-007-0805-y

Rasmussen, E., 1956. Faunistic and biological notes on marine invertebrates. III. The reproduction and larval development of some polychaetes from the Isefjord, with some faunistic notes. Biologiske Meddelelser udgivet af Det Kongelige Danske Videnskabernes Selskab, 23: 1-84.

Rasmussen, E., 1973. Systematics and ecology of the Isefjord marine fauna (Denmark). Ophelia, 11: 1-495.

Rioja, E., 1917. Datos para el conocimiento de la fauna de Anélidos Poliquetos del Cantábrico. Trabajos del Museo Nacional de Ciencias Naturales, Serie Zoológica, 29: 1-111.

Rioja, E., 1931. Estudio de los poliquetos de la península Ibérica. Memorias de la Academia de Ciencias Exactas, Físicas y Naturales de Madrid. Serie de Ciencias Naturales, 2: 1471.

Rioja, E., 1935. Anélidos Poliquetos procedentes de las campañas del Instituto Español de Oceanografía. Trabajos del Instituto Español de Oceanografia, 13: $1-44$.

Rizzo, A. E. \& Amaral, A. C. Z., 2001. Spatial distribution of annelids in the intertidal zone in São Sebastião Channel, Brazil. Scientia Marina, 65(4): 323-331. 
Roule, L., 1896. Résultats scientifiques de la campagne du «Caudan» dans le golf de la Gascogne. Annélides. Annales de l'Université de Lyon, 26: 439-471.

Rullier, F., 1963. Les annélides Polychètes du Bosphore, de la Mer de Marmara et de la Mer Noire, en relation avec celles de la Méditerranée. Rapports et Procès Verbaux des Réunions - Commission Internationale pour l'Exploration Scientifique de la Mer Méditerranée, 17(2): 161-260.

Rullier, F. \& Amoureux, L., 1970. Nouvelle contribution à l'étude de la faune des Annélides Polychètes du Maroc. Bulletin de la Société des Sciences Naturelles et Physiques du Maroc, 49(1/2): 109-142.

Rullier, F. \& Amoureux, L., 1979. Annélides polychètes. Campagne de la Calypso au large des côtes atlantiques de l'Amérique du sud (1961-1962). I. Résultats Scientifiques des Campagnes de la Calypso Fascicule XI. Annales de l'Institut Océanographique (Suppl. 55): 145-206.

Saint-Joseph, A. de, 1898. Les Annélides polychètes des côtes de France (Manche et Océan). Annales des Sciences naturelles (Zoologie) Série 8, 5: 209-464.

Saint-Joseph, A. de, 1906. Annélides Polychètes des Côtes de France (Océan et Côtes de Provence. Annales des Sciences Naturelles, (Zoologie) Série 9, 3: 145-260.

Salazar-Vallejo, S. I. \& Londoño-Mesa, M., 2004. Lista de especies y bibliografía de poliquetos (Polychaeta) del Pacífico Oriental Tropical. Anales del Instituto de Biología, Universidad Nacional Autónoma de México, Serie Zoología, 75(1): 9-97.

San Martín, G., Acero, M., Contonente, M. \& Gómez, J., 1982. Una colección de Anélidos Poliquetos de las costas Mediterráneas Andaluzas. In: J. Ros \& F.X. Niell (eds.). Actas II Simposio Ibérico de Estudios del Bentos Marino, Barcelona, 3: 171-182.

San Martín, G., Estape, S., García-Ocejo, A., Gómez, C. \& Jiménez, P., 1990. Estudio de la taxocenosis de anélidos poliquetos de rizomas de Posidonia oceanica en las costa de Almería. Boletín Instituto Español de Oceanografía, 6(1): 41-58.

San Martín, G. \& Viéitez, J.M., 1984. Anélidos poliquetos de los rizomas de Posidonia oceanica en las costas del Cabo de Palos (Murcia, España). In: C.F. Boudouresque \& A. Jeudy (eds.). International Workshop on Posidonia oceanica Beds. GIS Posidonie Publications, 1: 149-157.

Sardá, R., 1984. Estudio sobre la fauna de Anélidos Poliquetos de las zonas mediolitoral e infralitoral en la región del Estrecho de Gibraltar. Tesis Doctoral. Universidad de Barcelona, Barcelona. 901 pp. (inédita).

Sardá, R., 1986. Contribución al conocimiento de las poblaciones anelidianas infaunales de la Costa Catalana. Publicaciones Departamento Zoología, Universidad Barcelona, 12: 27-36.
Sardá, R., 1991. Polychaete communities related to plant covering in the mediolittoral and infralittoral zones of the Balearic Islands (Western Mediterranean). Marine Ecology, 12(4): 341-360. doi: 10.1111/j.1439-0485. 1991.tb00263.x

Sardá, R., Martin, D., Pinedo, S., Dueso, A. \& Cardell, M. J., 1995. Seasonal dynamics of shallow soft-bottom communities in western Mediterranean. In: A. Eleftheriou, A. D. Ansell \& C.J. Smith, (eds.). Biology and Ecology of Shallow Coastal Waters, Olsen \& Olsen, Fredensborg:191-198.

Sars, M., 1851. Beretning om en i Sommeren 1849 foretagen zoologisk Reise i Lofoten og Finmarken. $N y t$ Magazin for Naturvidenskaberne, 6: 121-211.

Schmarda, L. K., 1861. Neue wirbellose Thiere beobachtet und gesammelt auf einer Reise um die Erde 1853 bis 1857. 1. Band: Turbellarien, Rotatorien und Anneliden. Wihelm Engelmann, Leipzig, 164 pp.

Serrano, A., 2002. Ecología de las poblaciones de poliquetos del entorno de la Isla de Mouro (Santander, Mar Cantábrico). Tesis Doctoral. Universidad Autónoma de Madrid. Madrid. 439 pp. (inédita).

Serrano, A., Sánchez, F. \& García-Castrillo, G., 2006. Epibenthic communities of trawlable grounds of the Cantabrian Sea. Scientia Marina, 70S1: 149-159.

Simboura, N. \& Nicolaidou, A., 2001. The Polychaetes (Annelida, Polychaeta) of Greece: checklist, distribution and ecological characteristics. National Centre of Marine Research, Monographs on Marine Sciences, Attiki, Greece, 4: 1-115.

Tena, J., 1992. Anélidos Poliquetos del Antepuerto de Valencia: Ecología y aspectos tróficos. Tesis de Licenciatura. Universitat de València. València. 270 pp. (inédita).

Tena, J., 1996. Faunística y ecología de los Anélidos Poliquetos de los fondos de sustrato duro del Archipiélago de las Chafarinas (S. W Mar de Alborán). Tesis Doctoral. Universidad de Valencia, Valencia. 549 pp. (inédita).

Tenore, K. R., Cal, R.M., Hanson, R. B., López-Jamar, E., Santiago, G. \& Tietjen, J. H., 1984. Coastal upwelling off the Rias Bajas, Galicia, NW Spain II. Benthic studies. Rapport et Procès-verbaux de Réunions. Conseil International pour l'Explotation de la Mer, 183: 91-100.

Thomassin, B., 1970. Contribution a l'étude des polychètes de la région de Tulear (S.W. de Madagascar), III. Sur les Capitellidae des sables corallines. Recueil des Travaux de la Station Marine d'Endoume (suppl. 10): 71-101.

Torres-Gavilá, F. J., 1989. Anélidos Poliquetos y comunidades bentónicas de la desembocadura del río Segura (Alicante, Mediterráneo Occidental). Tesis de Licenciatura. Universitat de València. València. 194 pp. (inédita). 
Torres-Gavilá, F. J., 2008. Estudio faunistico, ecológico y ambiental de la fauna de Anélidos Poliquetos de sustratos sueltos de las islas Chafarinas (Mar de Alborán S.W.Mediterráneo). Tesis Doctoral. Universidad de Valencia, Valencia. 720 pp. (inédita).

Verrill, A. E., 1873. Report upon the invertebrate animals of Vineyard Sound and the adjacent waters, with an account of the physical characters of the region. Report of the United States Fisheries Commission for 1871-1872: 295-778.

Verrill, A. E., 1874a. On the results of recent dredging expeditions on the coast of New England. American Journal of Science, 7: 498-505.

Verrill, A. E., 1874b. Explorations of Casco Bay by the U.S. Fish Commission, in 1873. Proceedings of the American Association for the Advancement of Science, 22: 340-395.

Viéitez, J. M., 1976. Ecología de Poliquetos y Moluscos de la playa de Meira (Ría de Vigo). I. Estudio de las comunidades. Investigaciones Pesqueras, 40(1): 223-248.

Viéitez, J. M., 1977. Primera cita para España de la especie Phoronis psammophila Cori. Boletín de la Real Sociedad Española de Historia Natural (Sección Biológica), 75: 243-248.

Viéitez, J. M., 1978. Comparación ecológica de dos playas de las Rías de Pontevedra y Vigo. Tesis Doctoral. Universidad Complutense de Madrid, Madrid. 273 pp. (inédita).

Viéitez, J. M., 1979. Ecología comparada de dos playas de la Rías de Pontevedra y Vigo, Fundación Juan March, Serie Universidad, 91: 1-46.

Viéitez, J. M. \& López Cotelo, I., 1982. Estudio faunístico de la playa de Barra (Ría de Vigo). Primeros resultados Oecologia aquatica, 6: 37-40.

Villalba, A. \& Viéitez, J. M., 1985. Estudio de la fauna de anélidos poliquetos del substrato rocoso intermareal de una zona contaminada de la Ría de Pontevedra (Galicia). Cahiers de Biologie Marine, 26: 359-377.

Villarroya, I., 2005. Anélidos Poliquetos y comunidades bentónicas del yacimiento submarino de Sierra Helada (Alicante). Trabajo de Investigación (DEA). Universidad de Valencia. Valencia. 160 pp. (inédita).

Warren, L. M., 1976. A review of the genus Capitella (Polychaeta Capitellidae). Journal of Zoology, London, 180: 195-209. doi: 10.1111/j.1469-7998.19 76.tb04673.x

Warren, L. M., 1979. Mediomastus fragilis Rasmussen (Polychaeta: Capitellidae). A. Species newly recorded from British waters. Journal of the Marine Biological Association of the United Kingdom, 59: 757-760. doi: http://dx.doi.org/10.1017/S0025315400045732

Warren, L. M., 1991. Problems in capitellid taxonomy. The genera Capitella, Capitomastus and Capitellides (Polychaeta). Ophelia (Suppl. 5): 275-282.
Watanabe, T. \& Shouzen, K., 1999. Occurrence of Peresiella clymenoides Harmelin, Capitellidae (Annelida: Polychaeta) in Toyama Bay, Central Japan. Bulletin of Toyama Prefectural Fisheries Experiment Station, 11: 61-66

Webster, H. E., 1886. The Annelida Chaetopoda of New Jersey. Annual Reports of the New York State Museum of Natural History, 39: 128-159.

Wehe, T. \& Fiege, D., 2002. Annotated checklist of the polychaete species of the seas surrounding the Arabian Peninsula: Red Sea, Gulf of Aden, Arabian Sea, Gulf of Oman, Arabian Gulf. Fauna of Arabia, 19: 7-238.

Wesenberg-Lund, E., 1949. Polychaetes of the Iranian Gulf. In. K. Jessen \& R. Spärck, (eds.). Danish scientific investigations in Iran, 4: 247-400.

Zavodnik, D., Vidakovic, J. \& Amoureux, L., 1985. Contribution to the sediment macrofauna in the area of Rovinj (North Adriatic Sea). Cahiers de Biologie Marine, 26: 431-444.

Zenetos, A, Cinar, M. E., Pancuci-Papadopoulou, M. A., Harmelin, J. G., Furnari, G., Andaloro, F., Belou, N., Streftaris, N. \& Zibrowius, H., 2005. Annotated list of marine alien species in the Mediterranean with records of the worst invasive species. Mediterranean Marine Science, 6(2): 63-118. 
Appendix.- Summary of Capitellidae taxa from the Iberian Peninsula, Chafarinas, Balearic and Canary Islands with thoracic formula and diagrammatic representation (thorax and some abdominal segments) for genera or species.

Apéndice.- Resumen de de taxones de la familia Capitellidae de la Península Ibérica, Islas Chafarinas, Baleares y Canarias con fórmula setal torácica (tórax y algunos segmentos abdominales) para géneros o especies.

Legend / Leyenda:

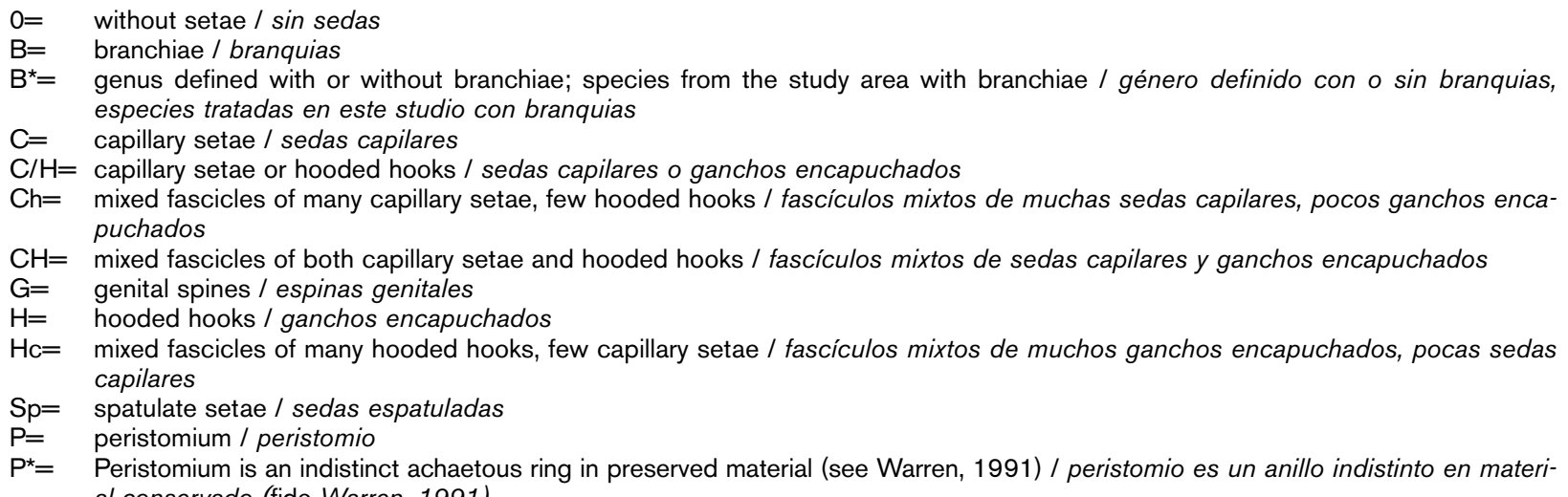

$\mathrm{P}^{*}=$ Peristomium is an indistinct achaetous ring in preserved material (see Warren, 1991) / peristomio es un anillo indistinto en material conservado (fide Warren, 1991)

$=$ prostomium / prostomio

\begin{tabular}{l|l}
$\mathrm{C}$ & $\mathrm{H}$ \\
$\mathrm{C}$ & $\mathrm{H}$
\end{tabular}$\quad$ = boundary between thorax and abdomen / límite entre tórax y abdomen

Family CAPITELLIDAE Grube 1862

Genus Capitella Blainville, 1828

Capitella capitata (Fabricius, 1780) sensu lato

$P^{*}+\frac{6 \mathrm{C}+\mathrm{C} / \mathrm{H} / \mathrm{CH}+2(\mathrm{G} / \mathrm{H})}{6 \mathrm{C}+\mathrm{C} / \mathrm{H} / \mathrm{CH}+2 \mathrm{H}}$

Thoracic region

Capitella giardi (Mesnil, 1897)

$$
P^{*}+\frac{6 C+H+2 G}{6 C+3 H}
$$

\begin{tabular}{|l|l|l|l|l|l|l|l|l|l|l|l|}
\hline & $\mathrm{C}$ & $\mathrm{C}$ & $\mathrm{C}$ & $\mathrm{C}$ & $\mathrm{C}$ & $\mathrm{C}$ & $\mathrm{H}$ & $\mathrm{G}$ & $\mathrm{G}$ & $\mathrm{H}$ & \\
$\mathrm{C}$ & $\mathrm{C}$ & $\mathrm{C}$ & $\mathrm{C}$ & $\mathrm{C}$ & $\mathrm{C}$ & $\mathrm{H}$ & $\mathrm{H}$ & $\mathrm{H}$ & $\mathrm{H}$ & - \\
\hline 1 & 2 & 3 & 4 & 5 & 6 & 7 & 8 & 9
\end{tabular}

Capitella minima Langerhans, 1880

$$
\begin{aligned}
& P+\frac{4 C+3 H+2 G}{4 C+5 H} \\
& P+\frac{3 C+4 H+2 G}{3 C+6 H}
\end{aligned}
$$

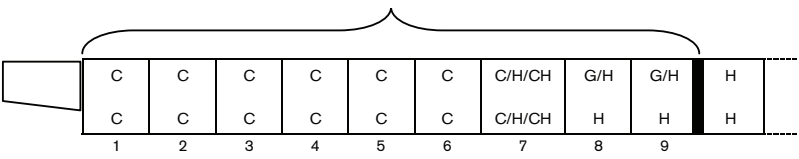$$
\begin{array}{|l|l|l|l|l|l|l|l|l|l|l|l|l|}
\hline & & C & C & C & C & H & H & H & G & G & H \\
\hline & & C & C & C & C & H & H & H & H & H & H \\
\hline
\end{array}
$$

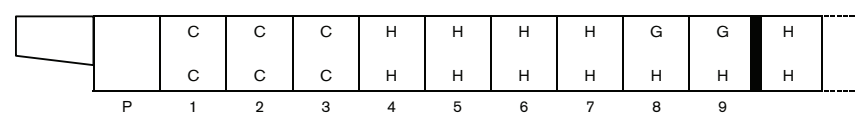


Genus Dasybranchus Grube, 1850

Dasybranchus caducus (Grube, 1846)

Dasybranchus gajolae Eisig, 1887

$$
P+\frac{13 C}{13 C}
$$

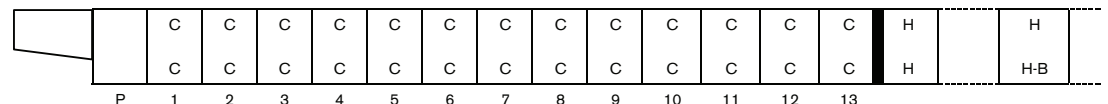

Genus Heteromastus Eisig, 1887

Heteromastus filiformis (Claparède, 1864)

$$
P+\frac{5 C+6 H}{5 C+6 H}
$$

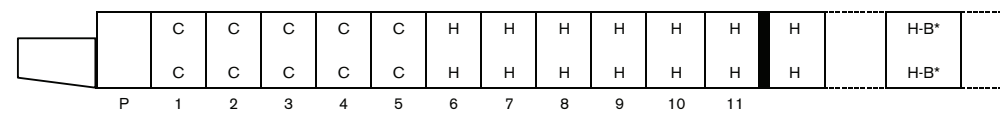

Leiocapitella Hartman, 1947

Leiocapitella glabra Hartman, 1947

$$
P+\frac{14 C}{0+12 C+1 H}
$$

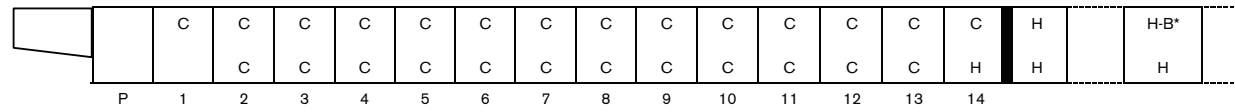

Genus Leiochrides Augener, 1914

Leiochrides cf. africanus Augener, 1918

$$
P+\frac{12 C}{12 C}
$$

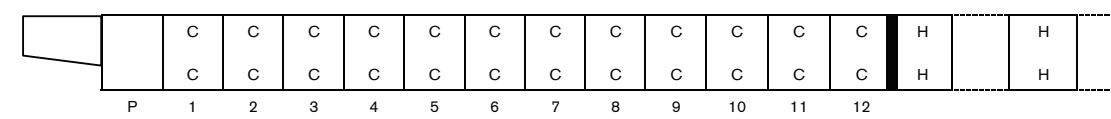

Genus Mastobranchus Eisig, 1887

Mastobranchus trinchesii Eisig, 1887

$$
P+\frac{9 C+2(C / H)}{0 / 1 C+8 C+2(C / H)}
$$

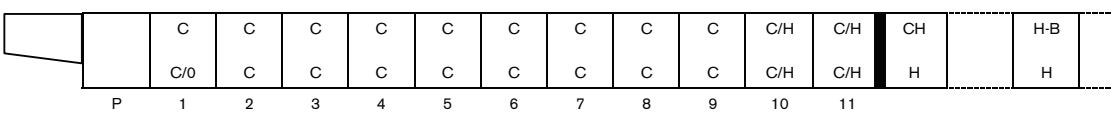

Genus Mediomastus Hartman, 1944

Mediomastus fragilis Rasmussen, 1973

Mediomastus capensis Day, 1961

$$
P+\frac{4 C+6 H}{4 C+6 H}
$$

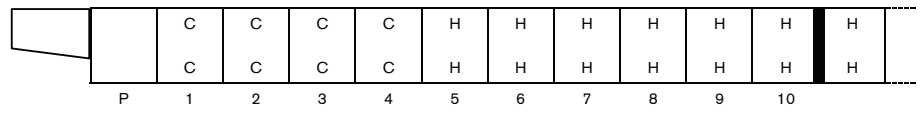

Genus Neopseudocapitella Rullier y Amoureux, 1979

Neopseudocapitella brasiliensis Rullier y Amoureux, 1979

$$
P+\frac{11 C+3(C h)}{0+10 C+3 H}
$$




Genus Notomastus Sars, 1851

Notomastus aberans Day, 1957

Notomastus agassizii Mclntosh, 1885

Notomastus exsertilis Saint-Joseph, 1906

Notomastus formianus Eisig, 1887

Notomastus latericeus Sars 1851

Notomastus lineatus Claparède, 1870

Notomastus profundus Eisig. 1887

$$
P+\frac{11 C}{1 C / 0+10 C}
$$

\begin{tabular}{|c|c|c|c|c|c|c|c|c|c|c|c|c|c|c|c|}
\hline & & $C$ & $C$ & $C$ & $C$ & $C$ & $C$ & $C$ & $C$ & $C$ & $C$ & $C$ & $H \cdot-B^{*}$ & & $\begin{array}{c}H \cdot B^{*} \\
\\
\end{array}$ \\
\hline
\end{tabular}

Genus Peresiella Harmelin, 1968

Peresiella clymenoides Harmelin, 1968

$$
P+\frac{3 C+8 S p}{0+2 C+8 S p}
$$

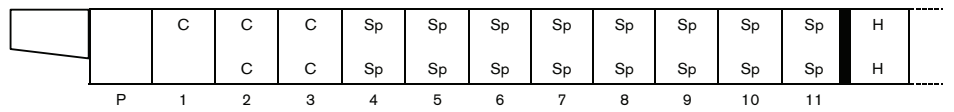

Genus Pseudocapitella Fauvel, 1913

Pseudocapitella incerta Fauvel, 1913

$$
P+\frac{17 C}{0+13 C+3 H}
$$

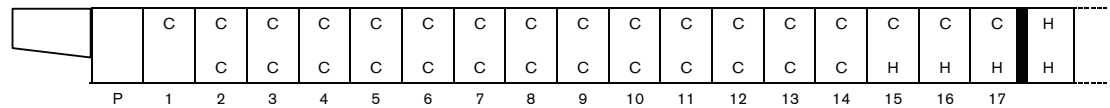

Pseudocapitella incerta aberrans Amoureux, 1972

$$
P+\frac{16 C}{0+12 C+3 H}
$$

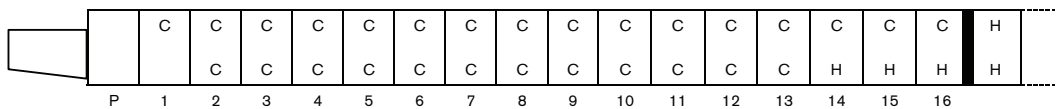

Genus Pseudoleiocapitella Harmelin, 1964

Pseudoleiocapitella fauveli Harmelin, 1964

$$
P+\frac{12 C}{0+9 C+2 H}
$$

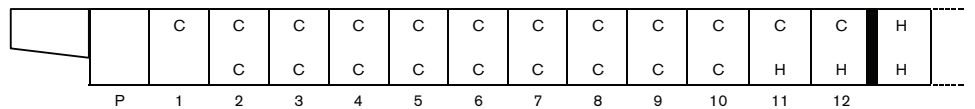

Genus Pseudomastus Capaccioni-Azzati y Martín, 1992

Pseudomastus deltaicus Capaccioni-Azzati y Martín, 1992

$$
P+\frac{12 C}{0+9 C+2 H}
$$

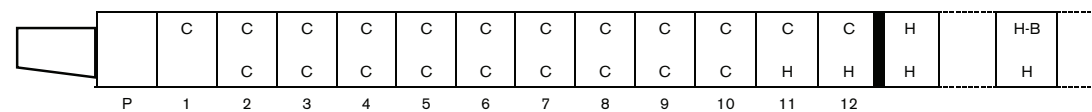

